\title{
New Insights into the Lactate Shuttle: Role of MCT4 in the Modulation of the Exercise Capacity.
}

\author{
Sara Bisetto \\ Thomas Jefferson University \\ Megan C Wright \\ Arcadia University \\ Romana A Nowak \\ The University of Illinois \\ Angelo C Lepore \\ Thomas Jefferson University \\ Eollow this and additional works at: https://jdc.jefferson.edu/pacbfp \\ Telvir S Khurana \\ ivenstityf Qhe earnlayltzayjiaommons \\ Let us know how access to this document benefits you
}

See next page for additional authors

\section{Recommended Citation}

Bisetto, Sara; Wright, Megan C; Nowak, Romana A; Lepore, Angelo C; Khurana, Tejvir S; Loro, Emanuele; and Philp, Nancy J, "New Insights into the Lactate Shuttle: Role of MCT4 in the Modulation of the Exercise Capacity." (2019). Department of Pathology, Anatomy, and Cell Biology Faculty Papers. Paper 291.

https://jdc.jefferson.edu/pacbfp/291

This Article is brought to you for free and open access by the Jefferson Digital Commons. The Jefferson Digital Commons is a service of Thomas Jefferson University's Center for Teaching and Learning (CTL). The Commons is a showcase for Jefferson books and journals, peer-reviewed scholarly publications, unique historical collections from the University archives, and teaching tools. The Jefferson Digital Commons allows researchers and interested readers anywhere in the world to learn about and keep up to date with Jefferson scholarship. This article has been accepted for inclusion in Department of Pathology, Anatomy, and Cell Biology Faculty Papers by an authorized administrator of the Jefferson Digital Commons. For more information, please contact: JeffersonDigitalCommons@jefferson.edu. 


\section{Authors}

Sara Bisetto, Megan C Wright, Romana A Nowak, Angelo C Lepore, Tejvir S Khurana, Emanuele Loro, and Nancy J Philp 


\section{Article}

New Insights into the Lactate Shuttle: Role of MCT4 in the Modulation of the Exercise Capacity

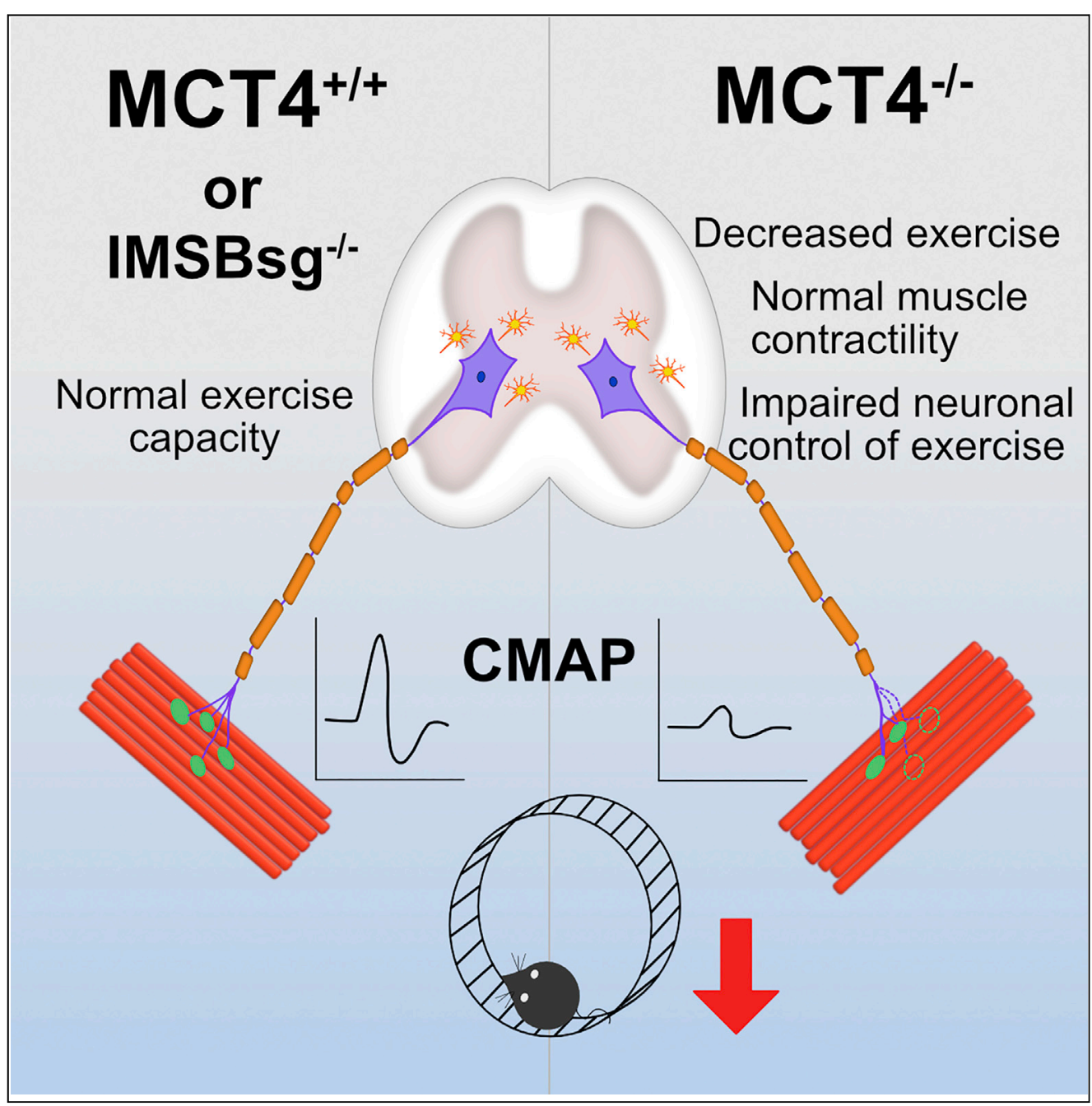

Sara Bisetto,

Megan C. Wright,

Romana A.

Nowak, Angelo C.

Lepore, Tejvir S.

Khurana,

Emanuele Loro,

Nancy J. Philp

eloro@pennmedicine.upenn.

edu (E.L.)

nancy.philp@jefferson.edu

(N.J.P.)

HIGHLIGHTS

MCT4 facilitates lactate

efflux from the cells and is

highly expressed in

muscles fibers

$\mathrm{MCT}^{-1-}$ mice are

exercise intolerant but

there is no impact on

muscle physiology

Action Potential

conduction is impaired in the $\mathrm{MCT}_{4}{ }^{-1}$ mice and

NMJs are disrupted

MCT4 and lactate efflux

play a role in the

maintenance of a

functional motor unit 
Article

\title{
New Insights into the Lactate Shuttle: Role of MCT4 in the Modulation of the Exercise Capacity
}

\author{
Sara Bisetto, ${ }^{1}$ Megan C. Wright, ${ }^{2}$ Romana A. Nowak, ${ }^{3}$ Angelo C. Lepore, ${ }^{4}$ Tejvir S. Khurana, ${ }^{5}$ Emanuele Loro, ${ }^{5, *}$ \\ and Nancy J. Philp ${ }^{1,6, *}$
}

\begin{abstract}
SUMMARY
Lactate produced by muscle during high-intensity activity is an important end product of glycolysis that supports whole body metabolism. The lactate shuttle model suggested that lactate produced by glycolytic muscle fibers is utilized by oxidative fibers. MCT4 is a proton coupled monocarboxylate transporter preferentially expressed in glycolytic muscle fibers and facilitates the lactate efflux. Here we investigated the exercise capacity of mice with disrupted lactate shuttle due to global deletion of MCT4 (MCT4 ${ }^{-/-}$) or muscle-specific deletion of the accessory protein Basigin (iMSBsg ${ }^{-/}$). Although $\mathrm{MCT4}^{-1-}$ and $\mathrm{iMSBsg}^{-1-}$ mice have normal muscle morphology and contractility, only $\mathrm{MCT4}^{-/}$ mice exhibit an exercise intolerant phenotype. In vivo measurements of compound muscle action potentials showed a decrement in the evoked response in the $\mathrm{MCT}^{-/-}$mice. This was accompanied by a significant structural degeneration of the neuromuscular junctions (NMJs). We propose that disruption of the lactate shuttle impacts motor function and destabilizes the motor unit.
\end{abstract}

\section{INTRODUCTION}

A hallmark of high-intensity exercise is the increased production of lactate by muscles, which is coupled with an increase in lactate in circulation. Lactate was once considered a waste product of anaerobic glycolysis and the cause of muscle fatigue and decreased force generation (Hill et al., 1924). In the late 1980s, George Brooks (Brooks, 1986) proposed that lactate produced by fast-twitch muscle fibers through both aerobic and anaerobic glycolysis (Connett et al., 1990) is shuttled to oxidative fibers and to other organs via circulation, to fuel oxidative metabolism during exercise.

Lactate is transported across the plasma membrane by monocarboxylate transporters (MCTs), encoded by the SLC16A gene family (Adijanto and Philp, 2012). MCT1 (SLC16A1) and MCT4 (SLC16A3) are $\mathrm{H}^{+}$-coupled lactate transporters found in the sarcolemma of skeletal muscle (Bonen, 2001; Wilson et al., 1998). MCT1 has higher affinity for lactate (Poole and Halestrap, 1993) and is predominantly expressed in slow-twitch muscle fibers, where it facilitates the influx of lactate. MCT4 has lower affinity but higher capacity for lactate transport (Dimmer et al., 2000) and is expressed in fast twitch where it mediates lactate efflux. The direction of the flux is primarily controlled by the gradient of lactate and protons (Juel, 1997). To be trafficked to the plasma membrane, MCT1 and MCT4 need to assemble in the ER with an accessory protein called Basigin (Bsg or CD147) (Kirk et al., 2000), a member of the immunoglobulin superfamily. In Bsg knockout mice, MCTs are not trafficked to the plasma membrane but are targeted for degradation (Philp et al., 2003).

The model of the lactate shuttle as a mechanism to distribute high-energy metabolites between glycolytic and oxidative cells has been applied beyond skeletal muscle and exercise. It has been suggested that lactate is shuttled between astrocytes and neurons during neuronal stimulation (Pellerin et al., 1998). In the brain, it is not yet clear if the direction of the lactate shuttle is from astrocytes to neurons or vice versa. Multiple studies have tried to address the question, but the results are still inconclusive as discordant findings have been published (Díaz-García et al., 2017; Mangia et al., 2009).

In this study, we have examined the contribution of the lactate shuttle to exercise capacity of mice using a global Slc16a3 knockout (MCT4 ${ }^{-1-}$ ) and an inducible muscle specific Bsg knockout mouse (iMSBsg ${ }^{-1-}$ ) models. We performed in vivo and ex vivo analyses of exercise capacity, neuromuscular function, and morphometry in these mouse models. Surprisingly, our results revealed that disruption of lactate transport

\footnotetext{
${ }^{1}$ Department of Pathology, Anatomy and Cell Biology, Thomas Jefferson University, Philadelphia, PA 19107, USA

${ }^{2}$ Department of Biology, Arcadia University, Glenside, PA 19038, USA

Institute for Genomic Biology, University of Illinois, Urbana, IL 61801, USA

${ }^{4}$ Department of Neuroscience, Thomas Jefferson University, Philadelphia, PA 19107, USA ${ }^{5}$ Department of Physiology and Pennsylvania Muscle Institute, Perelman School of Medicine, University of Pennsylvania, Philadelphia, PA 19104, USA

6Lead Contact

*Correspondence: eloro@pennmedicine.upenn. edu (E.L.), nancy.philp@jefferson.edu (N.J.P.)

https://doi.org/10.1016/j.isci. 2019.11.041
} 

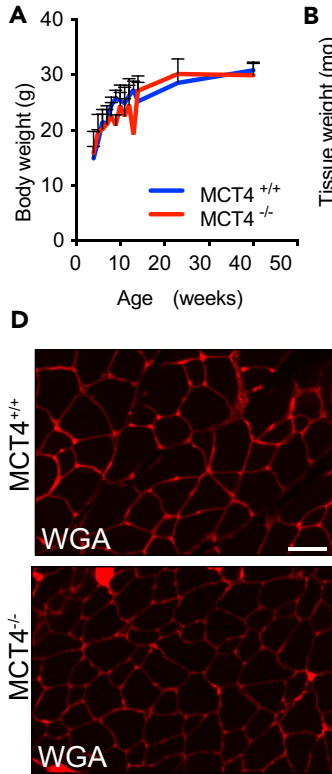

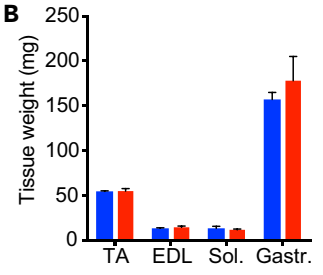

C

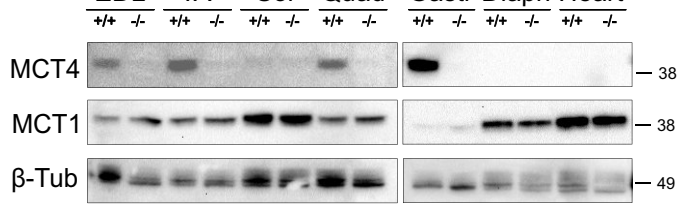

E

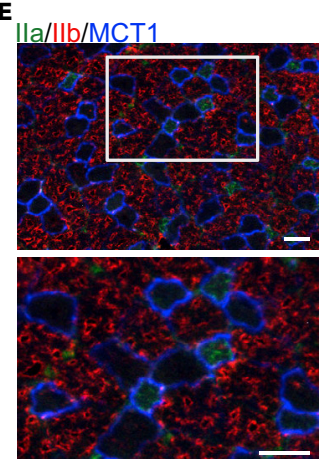

Figure 1. $\mathrm{MCT}^{-1-}$ Mice Have Normal Development

( $A$ and $B$ ) (A) Weight gain over 40 weeks of age and (B) muscle weight at 3 months of age in MCT4 ${ }^{+/+}$and MCT4 ${ }^{-/-}$mice ( $n=15$ per group). Values are mean and SEM.

(C) Western blot analysis of MCT4 and MCT1 expression in skeletal muscle and cardiac muscle. EDL, extensor digitorum longus; TA, tibialis anterior; Sol, soleus; Quad, quadricep; Gastr, gastrocnemius; Diaph, diaphragm.

(D) Representative immunostaining of EDL frozen sections from $\mathrm{MCT}^{-/-}$and $\mathrm{MCT}^{+/+}$mice labeled with WGA (left, red) and antibodies against MCT4 (middle, green) and MCT1 (right, green).

(E) Representative immunolabeling of EDL frozen sections with MCT1 (blue) and antibodies against type IIb (red) and Ila (green) fibers. Scale bar, $50 \mu \mathrm{m}$.

decreased exercise performance without altering muscle structure/function and was accompanied by a progressive structural and functional impairment of the neuronal component of the motor unit.

\section{RESULTS}

MCT4 $^{-1-}$ Mice Develop Exercise Intolerance

$\mathrm{MCT}^{-1-}$ mice were purchased from Taconic Biosciences. The animals were born at normal Mendelian ratio, with no apparent defects at birth. There were no developmental delays noted in $\mathrm{MCT}^{-1-}$ mice, as confirmed by normal growth curves and comparable body (Figure 1A) and muscle (Figure 1B) weights between $\mathrm{MCT4}^{+/+}$and $\mathrm{MCT}_{4}^{-/-}$mice.

MCT4 was previously shown to be expressed in fast-twitch muscle fibers (Bonen, 2001). In agreement with this we found by western blot analysis of muscle lysates from $\mathrm{MCT4}^{+/+}$mice that MCT4 was mainly expressed by glycolytic fast-twitch (EDL, TA, and quadriceps) and mixed (gastrocnemius) muscles. MCT4 was completely absent in muscles lysates prepared from $\mathrm{MCT}^{-1-}$ mice (Figure 1C). MCT1, on the other hand, was expressed in all muscle types, but at higher levels in oxidative slow-twitch muscles including soleus, diaphragm, and heart (Figure 1C). We performed immunofluorescent labeling on frozen sections of the extensor digitorum longus (EDL) and found MCT4 was localized at the sarcolemma in all the fibers of $\mathrm{MCT4}^{+/+} \mathrm{EDL}$ muscles, where it co-localized with wheat germ agglutinin (WGA). No MCT4 labeling was detected in frozen sections of EDLs from MCT4 ${ }^{-1-}$ mice (Figure 1D). Because the MCTs are bidirectional and can function for influx or efflux of lactate, we examined whether MCT1 expression was increased in the EDL for compensation. MCT1 immunofluorescence on EDLs cryosections showed that the transporter has a similar expression pattern from both $\mathrm{MCT}^{+/+}$and $\mathrm{MCT}^{-/-}$mice, where it was preferentially expressed in oxidative fiber (smaller sized) (Figure 1D). Immunofluorescence labeling of EDL cross sections labeled with antibodies detecting MCT1, type Ila MyHC (oxidative fibers), and type Ilb MyHC (glycolytic fibers) confirmed that the transporter is primarily expressed in Ila and IIx (identified as non-positive for either Ila or Ilb MyHC) fibers (Figure 1E). 

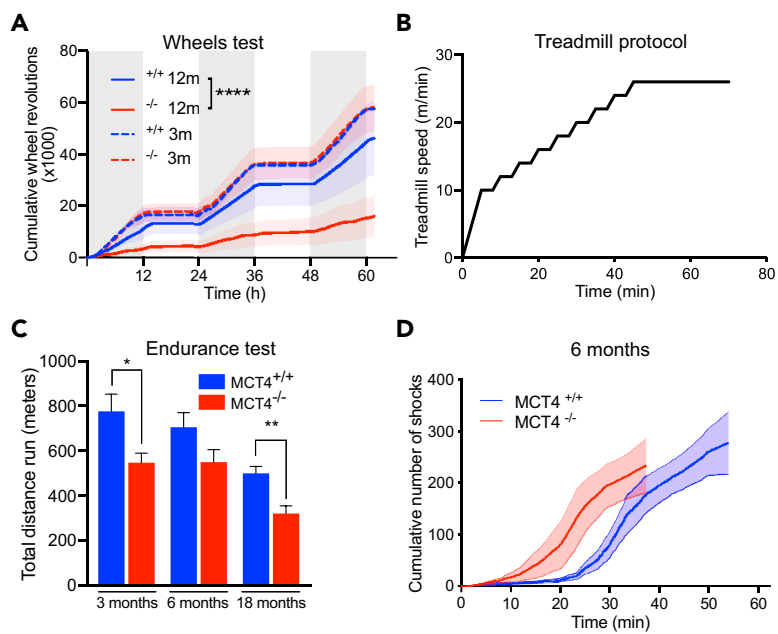

Figure 2. $\mathrm{MCT}^{-1-}$ Are Exercise Intolerant

(A) Voluntary wheel running, cumulative number of revolutions over $60 \mathrm{~h}$ of $\mathrm{MCT}^{-/-}$and $\mathrm{MCT}^{+/+}$mice, at 3 and

12 months of age $\left(n=3\right.$ each group, One way-ANOVA test, $\left.{ }^{\star \star \star *} p<0.0001\right)$.

(B) Treadmill protocol for endurance test at $5^{\circ}$ incline.

(C) Total distance reached in the endurance treadmill test by $\mathrm{MCT}^{+/+}$and $\mathrm{MCT4}^{-/-}$mice at 3, 6, and 18 months of age $\left(\mathrm{n}=10 \mathrm{MCT4}^{+/+}\right.$and $23 \mathrm{MCT}^{-/-}$at 3 months, $\mathrm{n}=10 \mathrm{MCT4}^{+/+}$and $15 \mathrm{MCT}^{-/-}$at 6 months, $\mathrm{n}=7 \mathrm{MCT}^{+/+}$and 8 $\mathrm{MCT}^{-1-}$ at 18 months, three independent groups. Student $\mathrm{t}$ test, ${ }^{*} \mathrm{p}<0.05,{ }^{* *} \mathrm{p}<0.001$ ).

(D) Representative traces of cumulative number of shocks over time during treadmill test of $\mathrm{MCT}^{-/-}$and $\mathrm{MCT}^{+/+}$mice at 6 months of age. All the data are rapresented as mean and SEM.

See also Figure S1.

To investigate whether alterations of lactate transport in $\mathrm{MCT}^{-1-}$ mice could affect normal exercise performance, we tested $\mathrm{MCT}_{4}{ }^{-/}$and $\mathrm{MCT}_{4}^{+/+}$mice with voluntary wheel running and forced endurance treadmill. Voluntary wheel activity was comparable between the two groups at 3 months of age but was reduced by 2.5 -fold in 12-month-old $\mathrm{MCT}^{-1-}$ mice (Figure $2 \mathrm{~A}$ ).

The endurance treadmill protocol was designed to gradually increase the running speed over time, up to $26 \mathrm{~m} / \mathrm{min}$, a speed at which oxygen consumption in C57BL/6 mice reaches approximately $80 \%$ of $\mathrm{VO}_{2} \mathrm{max}$ (high-intensity exercise) (Desai and Bernstein, 2001) (Figure 2B). At high-intensity, most of the energy to support muscle activity is derived from carbohydrates, resulting in the production and release of lactate from muscle fibers and the consequent increase in blood lactate. Consistently, blood glucose decreased by $\sim 30 \%$ after treadmill (Figure S2). Treadmill performance declined with age in both genotypes and, consistent with the wheel running results, $\mathrm{MCT}^{-1-}$ mice ran less compared with controls (Figure $2 \mathrm{C}$ ). $\mathrm{MCT4}^{-/-}$mice could reach the maximum speed but, unlike $\mathrm{MCT4}^{+/+}$, could not endure the high-intensity performance, as indicated by the quicker accumulation of electric shocks over time (Figure 2D). This suggests that, indeed, lack of MCT4 and disruption of muscle lactate efflux cause an age-dependent and exercise-intensity-dependent impairment of exercise capacity.

\section{Lack of MCT4 Does Not Affect the Intrinsic Properties of the EDL Muscle}

To determine if the exercise intolerance was due to structural alterations of $\mathrm{MCT}^{-1-}$ muscle fibers, we performed a histological analysis of EDL cross sections, looking for signs of degeneration, fibrosis, or fiber type switch. We focused on EDL muscles for a variety of reasons: first, EDL is highly engaged during locomotion in rodents; second, it expresses high levels of MCT4 and its fiber type composition is primarily fast; and finally, it can be easily and consistently dissected for functional studies.

H\&E staining showed normal fiber morphology with no signs of immune infiltrates, fibrotic tissue, or central nucleation (Figure 3A). Consistent with the normal muscle mass (Figure 1B), fiber size measured as minimum Feret diameter was comparable between genotypes both at 3 and 12 months of age (Figures 3B and 3 C). 

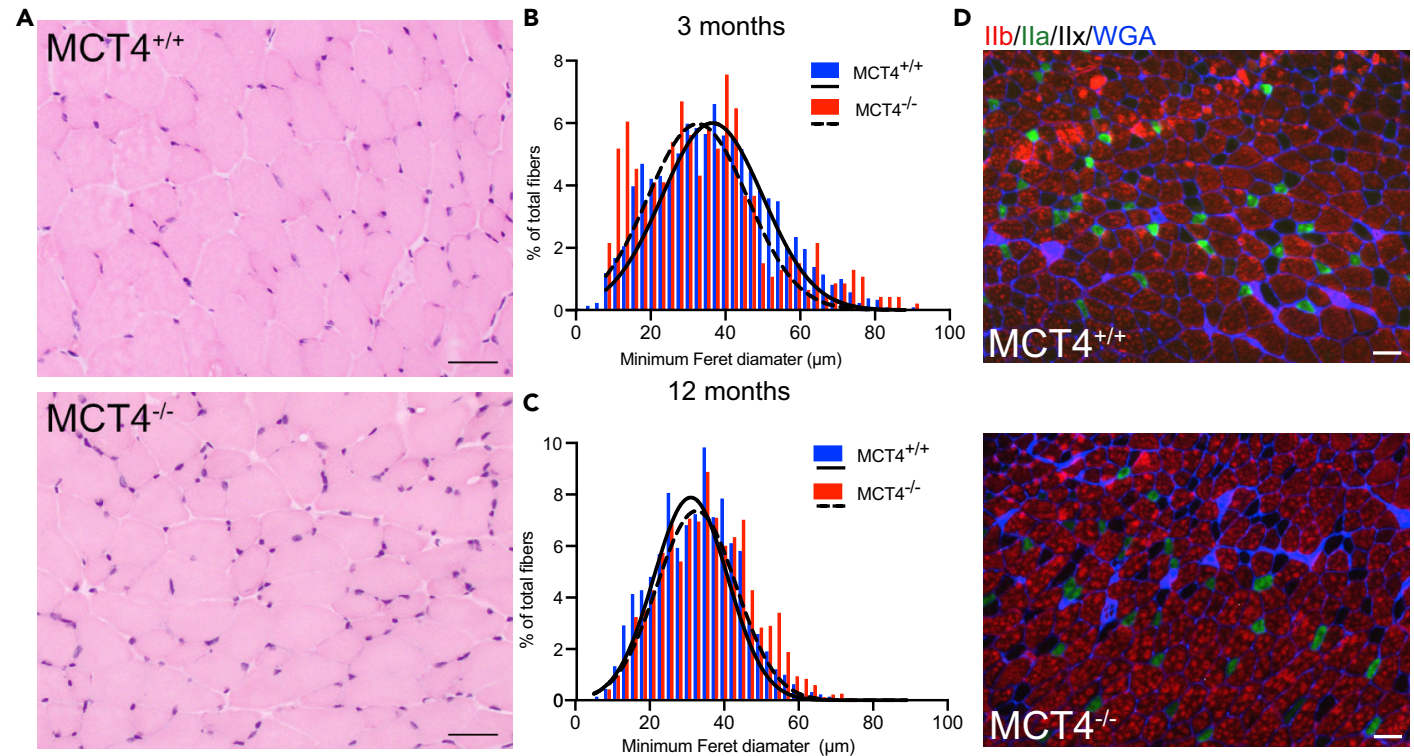
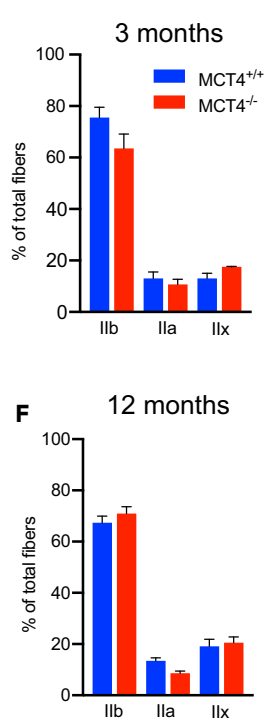

Figure 3. Skeletal Muscle Histology Is Not Affected by the Lack of MCT4

(A) Representative H\&E staining of EDL cryosections from $\mathrm{MCT}^{+/+}$and $\mathrm{MCT}^{-/-}$mice; scale bar, $50 \mu \mathrm{m}$.

( $B$ and C) Frequency histograms of fiber size of EDLs measured as minimum Feret diameter from MCT4 $4^{+/+}$and MCT4 ${ }^{-/-}$at $(B) 3$ and $(C) 12$ months $(n=5$ each group, counting all the fibers in the entire muscle section).

(D) Representative immunolabeling of $\mathrm{MCT}^{+/+}$and $\mathrm{MCT} 4^{-/-}$EDL sections with antibodies against type IIB and Ila fibers and laminin for sarcolemma. (E and F) Percentage of Ilb and Ila fiber types in EDLs from MCT4 ${ }^{+/+}$and MCT4 ${ }^{-/-}$at (E) 3 and (F) 12 months of age ( $n=5$ each group, two-way ANOVA test). All the data are rapresented as mean and SEM.

Changes in muscle metabolism are often correlated with changes in the proportion of glycolytic and oxidative fibers. To address this possibility, we immunolabeled EDL frozen sections with antibodies against the myosin heavy chain (MHC) isoforms (Figure 3D). The relative abundance of each fiber type was similar between genotypes at 3 and 12 months of age (Figures $3 E$ and $3 F$ ).

To determine if the exercise intolerance in $\mathrm{MCT}^{-/-}$mice could be caused by defects in muscle contractility, we performed an ex vivo assessment of strength and fatigability in isolated EDL muscles using field depolarization. This technique bypasses the neuronal and neuromuscular junction (NMJ) components and focuses specifically on measuring the contractile and metabolic properties of the muscle. Isometric twitch and tetanic forces, both absolute (Table S1) and after normalization by cross-sectional area (CSA) (Figures $4 \mathrm{~A}$ and $4 \mathrm{~B}$ ), were not different between $\mathrm{MCT}^{-1-}$ and $\mathrm{MCT}^{+/+}$. Similar optimal length $\left(\mathrm{L}_{0}\right)$, mass, and $\mathrm{CSA}$ were consistent with the tissue weight measurements (Figure 1B) and the histomorphometric analyses (Figures 3A-3C). EDL muscles from $\mathrm{MCT}^{+/+}$and $\mathrm{MCT}^{-/-}$mice had similar fatigability profiles (Figure $4 \mathrm{C}$ ), as determined by administering repeating submaximal tetanic contractions every second for $8 \mathrm{~min}$. The same trend was found in younger ( 3 months old) and older (18 months old) mice (data not shown). This was despite significant differences in the accumulation of intramuscular lactate, which was higher $(\sim 50 \%)$ in $\mathrm{MCT}^{-/-}$stimulated muscles compared with the unstimulated and $\mathrm{MCT}^{+/+}$conditions (Figure 4D). Therefore, intramuscular accumulation of lactate due to lack of MCT4 did not have an effect on muscle contractility. Because MCT4 is a proton-linked lactate transporter and contributes to the control of the intracellular $\mathrm{pH}$, we tested whether other $\mathrm{pH}$ modulators like carbonic anhydrases 2, 3, and 4 (Car2, Car3, Car4) or proton exchangers such as Mct 1 and $\mathrm{Na}^{+} / \mathrm{H}^{+}$exchanger (Nhe1) were overexpressed in the $\mathrm{MCT} 4^{-/}$muscle as a compensatory response. qPCR analysis of TA muscles from 6-month-old mice showed no difference in the expression of these genes (Figure 4E). Together, these data suggest that lactate accumulation in muscle does not alter muscle contractility or fatigability and therefore is not the main cause of the reduced exercise capacity of $\mathrm{MCT}^{-1-}$ mice.

\section{iMSBsg $^{-1-}$ Mice Have Normal Exercise Capacity}

To strengthen our finding and further investigate whether deletion of MCT4 in muscle could have any effect on exercise capacity, we generated mice with a skeletal muscle-specific deletion of Basigin (BSG). BSG is 

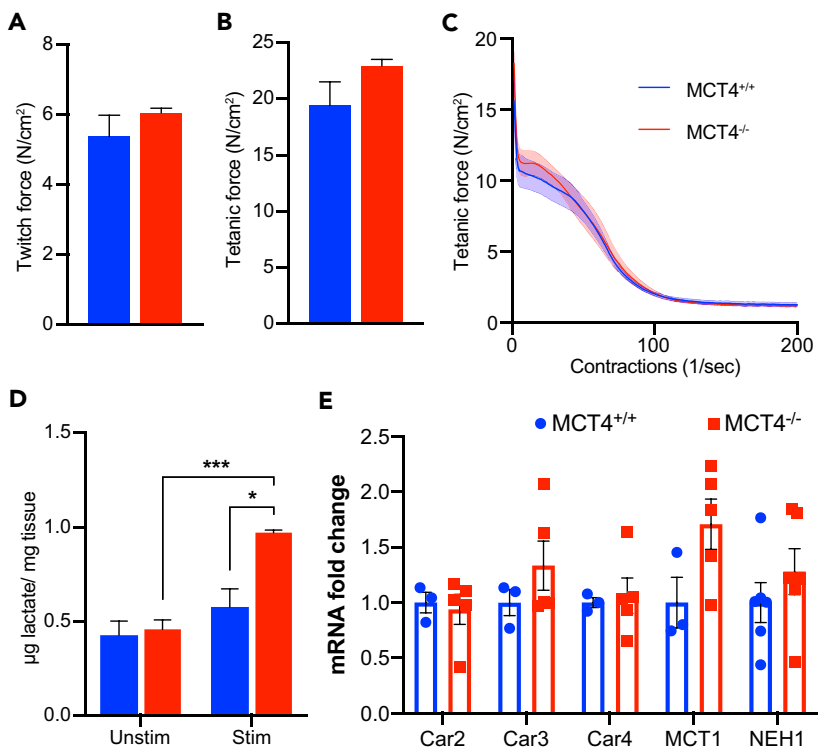

E

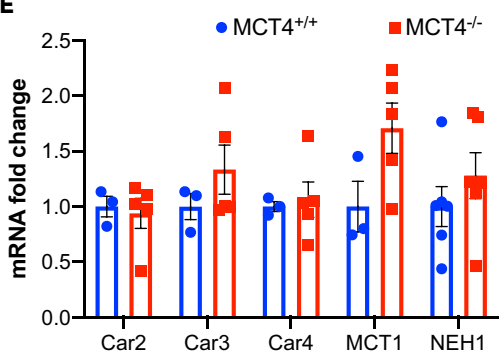

Figure 4. Lack of MCT4 Does Not Impair the Contractile Properties of the Skeletal Muscles ( $A$ and $B$ ) (A) EDL twitch and (B) tetanic forces generated ex vivo ( $n=4$ each group).

(C) EDL fatigue traces ( $n=4$ each group).

(D) Intramuscular lactate in unstimulated and stimulated gastrocnemius muscles ( $n=4$ each group, two-way ANOVA test, interaction: $p<0.01$, stimulation: ${ }^{* \star} p<0.001$, genotype: $\left.{ }^{\star} p<0.05\right)$.

(E) qPCR analysis of genes involved in $\mathrm{pH}$ control in TA muscles of 6 -month-old $\mathrm{MCT} 4^{+/+}$and $\mathrm{MCT} 4^{-1-}$ mice $\left(\mathrm{n}=3 \mathrm{MCT}^{+/+}\right.$and $5 \mathrm{MCT}^{-/-}$). All the data are rapresented as mean and SEM.

the accessory protein required for maturation and trafficking of both MCT1 and MCT4 to the sarcolemma. When Bsg is knocked out, MCT1 and MCT4 fail to traffic to the plasma membrane and are targeted for degradation. Specific deletion of Bsg in skeletal muscle was achieved by crossing Bsg Flox/Flox mice with the tamoxifen-inducible skeletal muscle-specific cre recombinase mice, Acta1-cre mice. The cre recombinases was induced when the mice reached 12 weeks of age to avoid possible developmental effects of the lack of BSG. Western blot analysis confirmed that Bsg was ablated from skeletal muscle of iMSBsg ${ }^{-1-}$ mice and that the cre-recombinase driven by Acta1 was not active in the cardiac muscle (Figure 5A). BSG, MCT1, and MCT4 were undetectable in lysates prepared from skeletal but not cardiac muscle.

Since tamoxifen can acutely affect cardiac muscle contractility (Asp et al., 2013), after the induction of the cre recombinase, we waited 3 weeks for the tamoxifen to be completely cleared from the system before testing the animals for exercise capacity. Using the forced treadmill test, we found that there was no significant difference in the total distance run by the $\mathrm{iMSBsg}^{-1-}$ mice compared with controls (Figure 5B). We then tested whether the complete disruption of the lactate shuttle had an effect on the contractile properties of the muscles by performing ex vivo measurements of force and fatigability in isolated EDLs. As for the $\mathrm{MCT}^{-1-}$ muscles, we did not detect any difference in maximal isometric force (Figures $5 \mathrm{C}$ and 5D) and fatigability (Figure 5E) between $\mathrm{iMSBsg}^{-1-}$ and control mice. Since the MCT4${ }^{-1-}$ presented a decrease in exercise capacity already at 3 months and we did not observe any difference between controls and $\mathrm{iMBsg}^{-/-}$, we did not investigate the performance of these animals at older ages. Overall, the studies on $\mathrm{iMSBsg}^{-1-}$ mice confirmed that the absence of MCT1 and MCT4 in the sarcolemma of skeletal muscle does not affect exercise capacity and muscle ex vivo contractile properties.

\footnotetext{
Loss of MCT4 Leads to Degeneration of the NMJ and Decreased Compound Muscle Action Potential Amplitude

In many neuropathies (Hill, 2003), e.g., Charcot-Marie-Tooth disease (CMT) and Amyotrophic Lateral Sclerosis (ALS), a progressive decline in muscle strength and exercise capacity is caused by pathologies of the motoneuron, nerves, and/or the NMJ rather than a primary defect in the muscle. We therefore investigated whether ablation of MCT4 could impact the structure and function of the motor unit components upstream of muscle.
} 
A

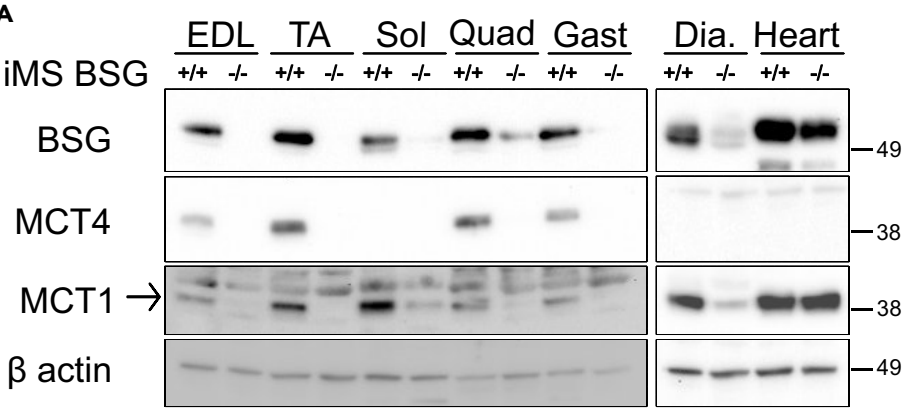

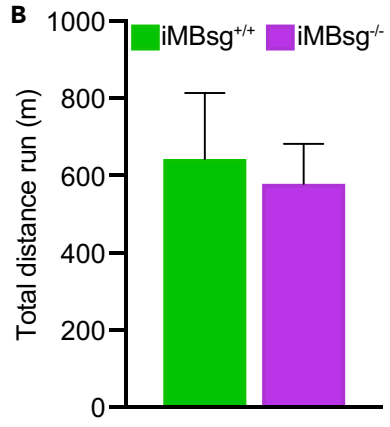
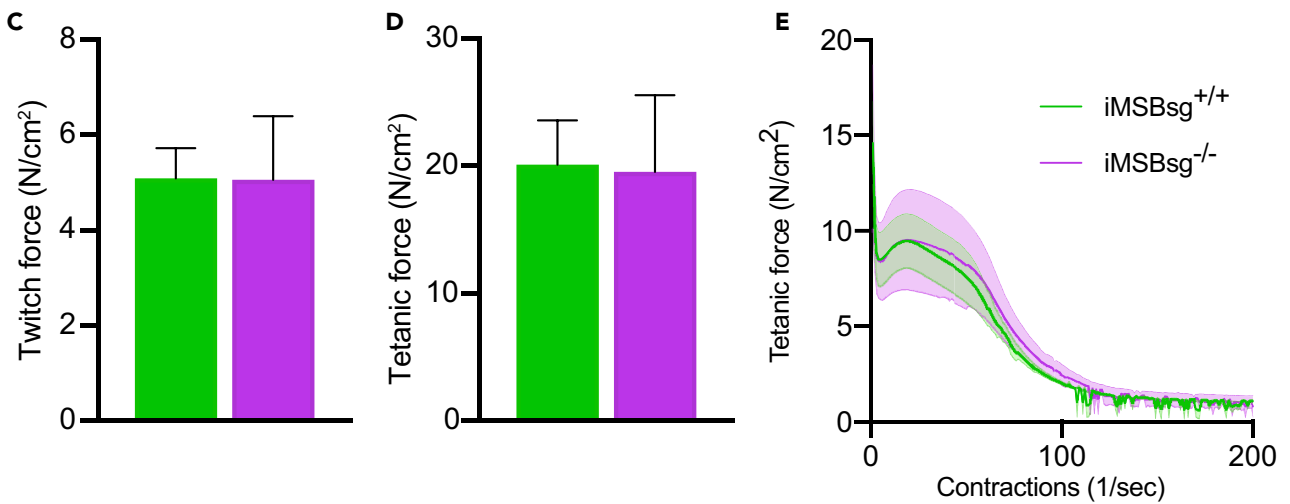

Figure 5. iMSBsg $^{-1-}$ Mice Do Not Show Exercise Intolerance

(A) Representative western blot analysis of BSG, MCT1, and MCT4 in skeletal muscles and cardiac muscle of iMSBsg ${ }^{-1-}$ and $\mathrm{MSBsg}^{+/+}$mice after tamoxifen or vehicle treatment.

(B) Total distance reached during the treadmill test by $\mathrm{iMSBsg}^{-/-}$and $\mathrm{MSBsg}^{+/+}$at 6 months of age ( $\mathrm{n}=5$ each group). Ex vivo EDL (C) twitch force, (D) tetanic force, and (E) fatigue traces ( $n=3$ each group). All the data are rapresented as mean and SEM.

See also Figure 52

It was previously reported that MCT4 is expressed in astrocytes in the Central Nervous System (CNS), particularly in the cortex and hippocampus of both rats and mice. qPCR analysis of Mct4 (SIc16a3) mRNA in spinal cord samples demonstrated the presence of the transcript, although the levels were 100 times lower than in skeletal muscle (Figure 6A). To determine the cellular distribution of MCT4-expressing cells in the spinal cord, we performed fluorescence in situ hybridization (FISH) on frozen sections from the lumbar spinal cord of $\mathrm{MCT}^{+/+}$mice and co-labeled with nuclear staining and GFAP (Glial fibrillary acidic protein), a marker for glial cells. The in situ hybridization showed widespread expression of Mct4 in both the gray and white matter (Figure 6B). Since we detected Mct4 expression in the spinal cord, we sought to test whether the lack of MCT4 affected the motor unit, beginning with the motoneuron. We used cresyl violet staining in lumbar spinal cord cryosections to detect the Nissl substance in neurons (Figure 6C). We did not detect any difference in the spinal cord histomorphological features between genotypes, with normal boundaries between white and gray matter. Motor neurons were identified as Nissl-positive cells with a clearly visible nucleus and a soma larger than $100 \mu \mathrm{m}$ and were counted in three separate sections of the lumbar spinal cord. The number of motoneurons was not different between $\mathrm{MCT}^{-/-}$and $\mathrm{MCT}^{+/+}$ (Figure 6D), suggesting that lack of MCT4 does not induce neuronal cell death.

We then performed a morphometric analysis of the sciatic nerve of 6-month-old $\mathrm{MCT}^{+/+}$and $\mathrm{MCT}^{-/-}$mice (Figure 6E) to determine if the lack of MCT4 altered the number, size, and/or myelination of axons. Toluidine blue staining of semithin sciatic nerve sections revealed no differences in the density of myelinated axons; however, we found a small but significant increase in axons diameters in $\mathrm{MCT}^{-1-}$ samples, driven by the increased occurrence of large axons (Figure 6F). The g-ratio (a measure of myelin thickness) was similar between genotypes.

The synapse between the motoneurons and the skeletal muscle is called neuromuscular junction (NMJ). Alterations in the NMJ are associated with poor motor performance and exercise intolerance. We then 

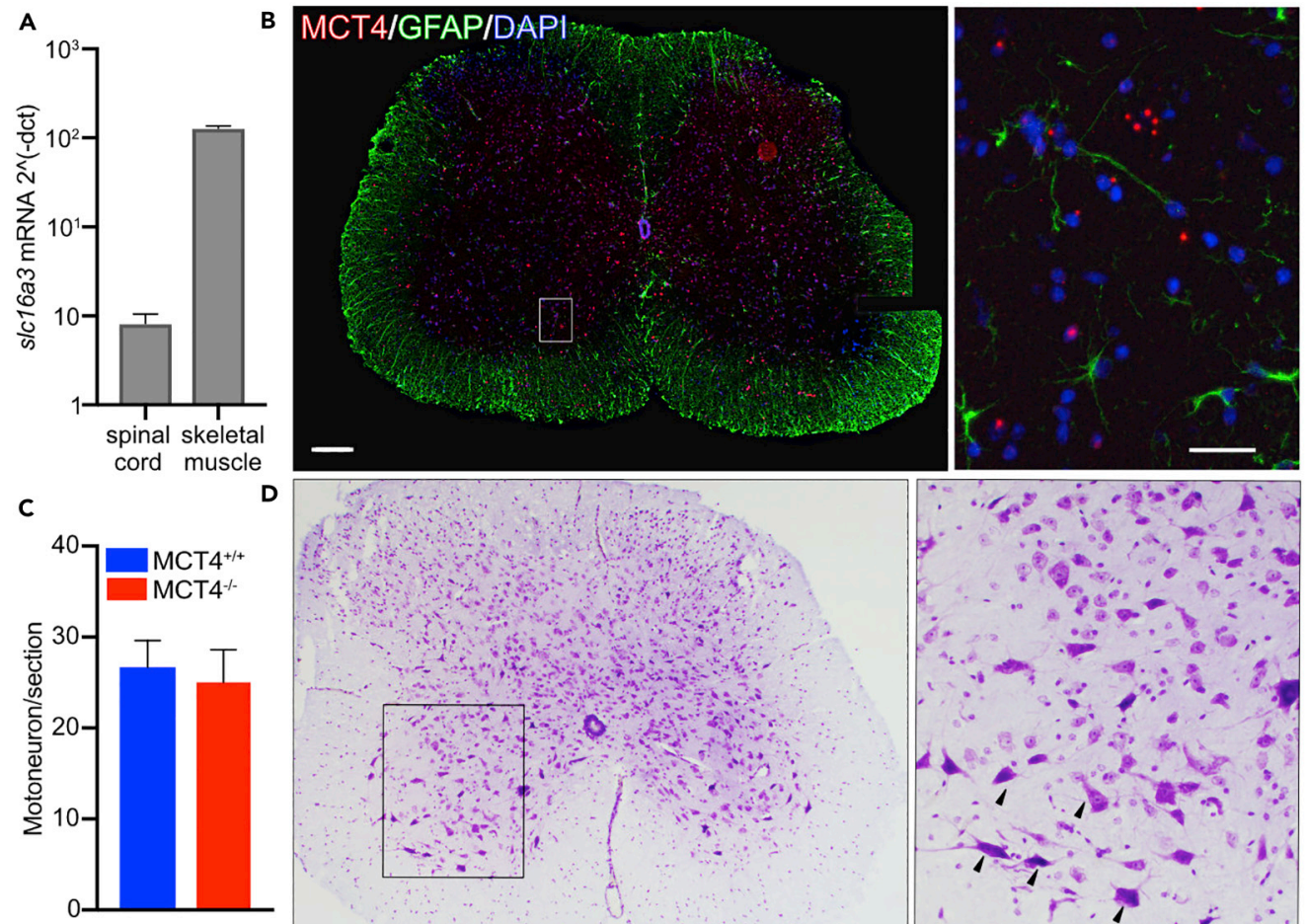

$\mathrm{D}$
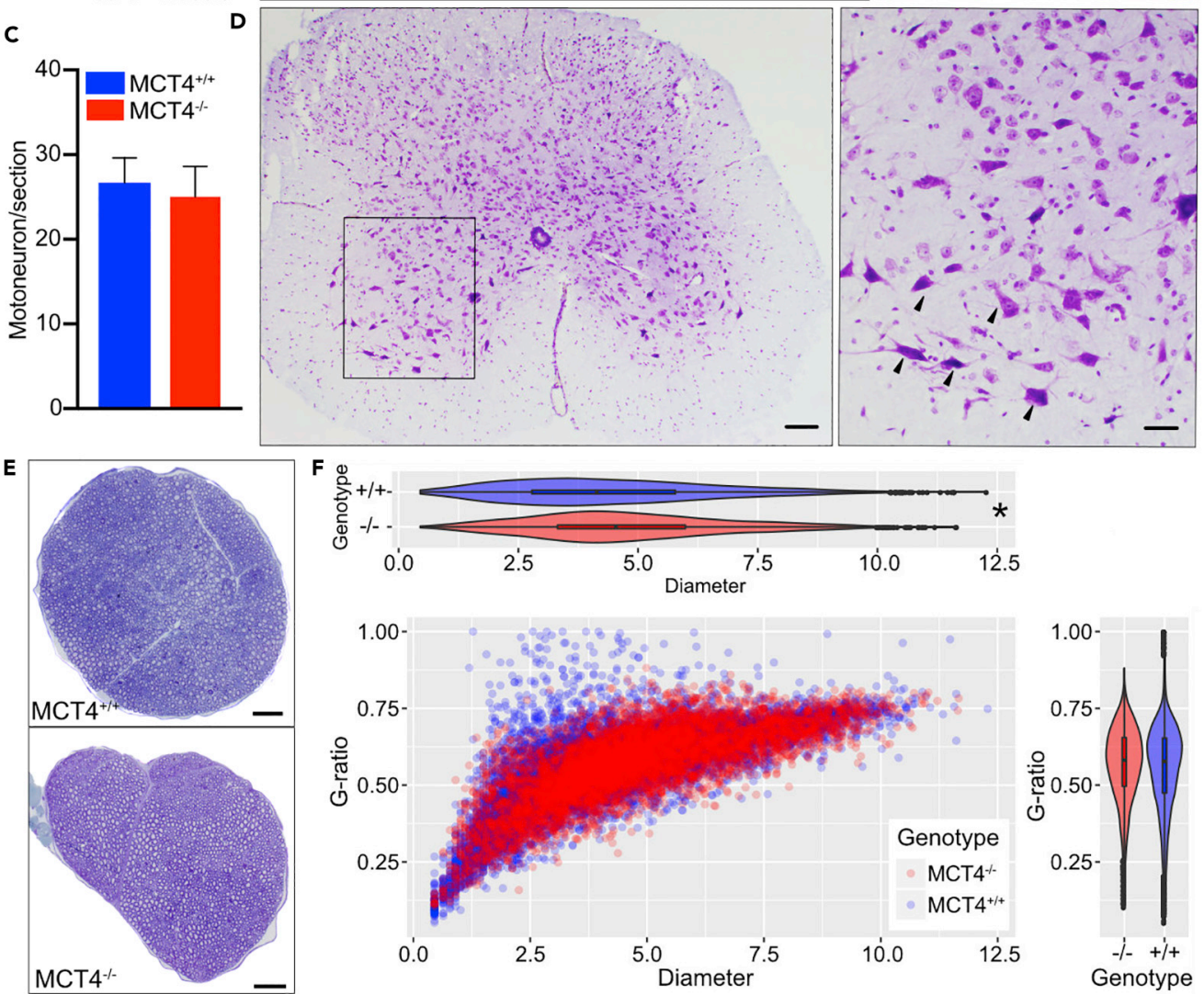

Figure 6. Lack of MCT4 Does Not Affect the Morphology of Motoneurons

(A) mRNA expression of Mct4 in spinal cord and skeletal muscle. Values are normalized to Rplp0 housekeeping gene.

(B) In situ hybridization of Mct4 mRNA (red) in lumbar spinal cord cryosections (10 $\mu \mathrm{m}$ thickness). Sections were co-labeled with nuclear DAPI staining (blue) and an antibody against GFAP (green). Scale bar, $200 \mu \mathrm{m}$; insert scale bar, $50 \mu \mathrm{m}$.

(C) Representative cresyl violet staining of lumbar spinal cord cryosections. Arrowheads in the enlarged insert indicate motoneurons in the ventral horn. Scale bar, $400 \mu \mathrm{m}$; insert scale bar, $200 \mu \mathrm{m}$.

(D) Quantification of the number of motoneurons in lumbar spinal cord sections $(25 \mu \mathrm{m})$ of $\mathrm{MCT}_{4}^{+/+}$and $\mathrm{MCT}^{-1-}$ mice ( $n=3$ animals each group, three sections per animal evaluated).

(E) Examples of toluidine blue staining of semithin sections of sciatic nerves $(0.5 \mu \mathrm{m}$ thickness). Scale bar, $200 \mu \mathrm{m}$.

(F) Morphometric analysis of axon diameter and g-ratio in $\mathrm{MCT}_{4}{ }^{-/-}$and $\mathrm{MCT4}^{+/+}$sciatic nerves $(\mathrm{n}=3$ each group, entire section was counted). ${ }^{\star} p<0.05$. All the data are rapresented as mean and SEM.

sought to understand if the lack of MCT4 affects the integrity of the NMJ. We assessed the morphology of the NMJ in EDL and soleus muscles by performing whole-mount immunolabeling of the pre-synaptic axons (SMI32 ab) and vesicles (SV2), as well as of the post-synaptic nicotinic acetylcholine receptors (alpha-bungarotoxin) (Figure 7A). Although we did not detect any significant difference in NMJ morphology in 


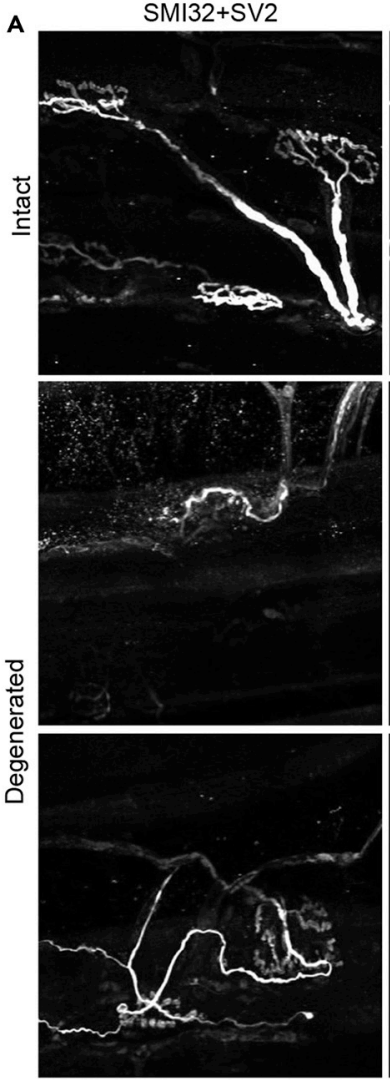

B

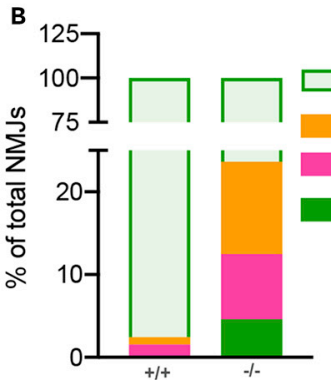

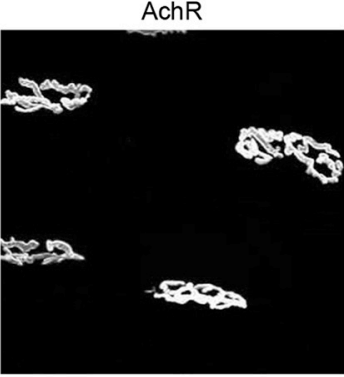
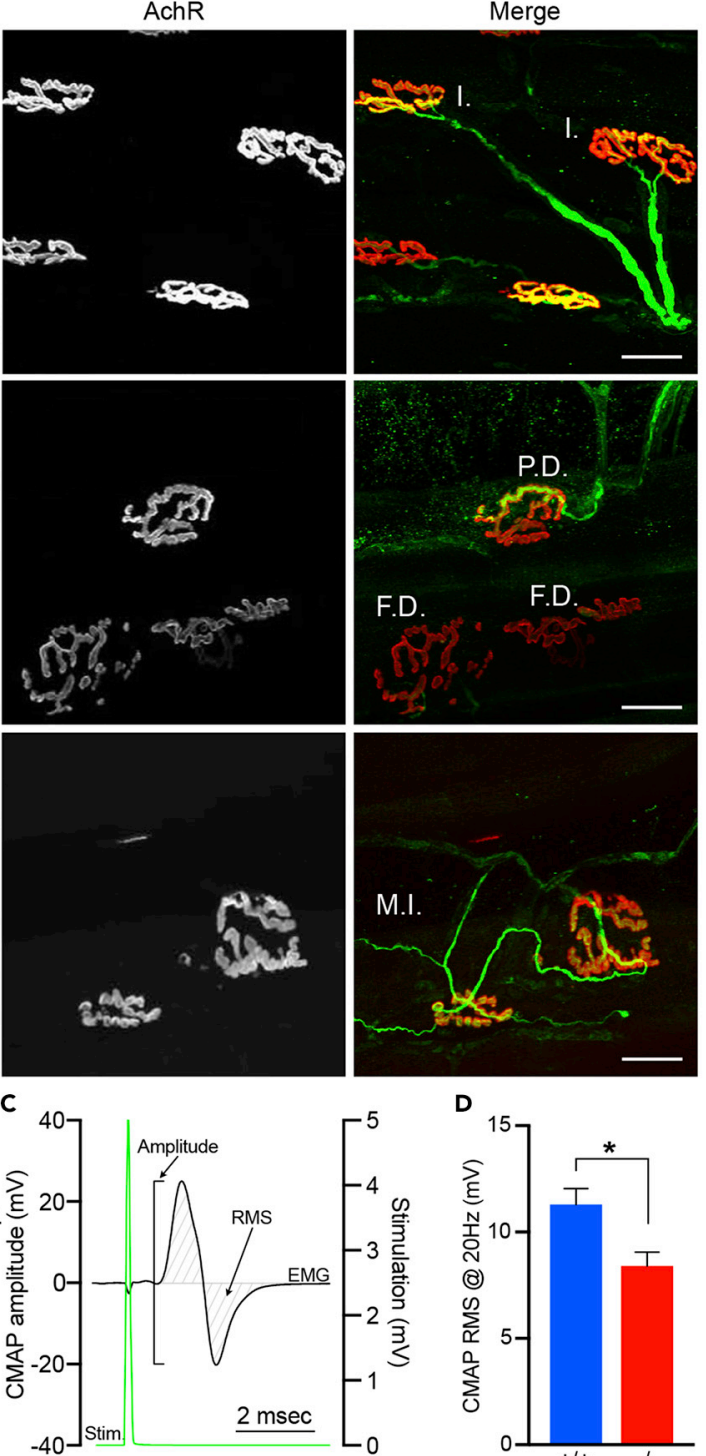

Figure 7. NMJ Morphology and CMAPs Are Impaired in MCT4 ${ }^{-1-}$ Mice

(A) Representative images of whole-mount immunohistochemistry of EDL muscles labeling motor axons (SMI32, green), pre-synaptic nerve terminals (SV2, green) and post-synaptic endplate (rhodamine-conjugated $\boldsymbol{\alpha}$-bungarotoxin, red). I., intact; P.D., partially denervated; F.D., fully denervated; M.I., multiple innervated.

(B) Quantification of intact and disrupted NMJs in EDLs of 12-month-old MCT4 ${ }^{-/-}$and MCT4 ${ }^{+/+}$( $\mathrm{n}=3$ each group

Chi-square, $\mathrm{p}<0.0001)$.

(C) Representative CMAP trace showing the electromyographic signal recorded from gastrocnemius muscle (black trace) upon stimulation of the sciatic nerve (green trace). Amplitude and RMS are indicated.

(D) CMAPs RMS (Root-Mean-Square) recorded at $20 \mathrm{~Hz}$ in 6-month-old MCT4 ${ }^{-/-}$and $\mathrm{MCT4}^{+/+}$mice ( $\mathrm{n}=4$ each group, Student $t$ test, $\left.{ }^{*} p<0.05\right)$. All the data are rapresented as mean and SEM.

See also Figure $\mathrm{S3}$

muscles from 6-month-old mice, the incidence of disrupted NMJs in EDL of 12-month-old $\mathrm{MCT4}^{-1-}$ mice was significantly higher ( $p<0.0001$, Chi-square test), with signs of denervation and re-innervation (Figure 7A). In 12-month-old $\mathrm{MCT}^{+/+}$muscles, only a minimum number of NMJs showed signs of partial denervation. Among the NMJs analyzed in $\mathrm{MCT}^{-1-}$ mice, $15 \%$ were partially or total denervated and $8 \%$ had terminal sprouting (Figures $7 \mathrm{~A}$ and $7 \mathrm{~B}$ ). The analysis of NMJs in the soleus muscles showed no difference between $\mathrm{MCT}^{-1-}$ and $\mathrm{MCT}^{+/+}$mice (Figure S3A), suggesting that the effects of MCT4 ablation are more evident in fast motor units. Analysis of the density of the acetylcholine receptors (AChRs) at the 
post-synaptic endplates showed a similar result, with an increase in the fragmentation of the NMJs of the 12-month-old $\mathrm{MCT}_{4}^{-1-}$ mice compared with the $\mathrm{MCT}^{+/+}$(Figures S3B and S3C). No difference in the integrity of the NMJs was detected in the 6-month-old mice.

To assess the functional activity of the motor unit, we performed in vivo compound muscle action potential (CMAP) studies on 6-month-old $\mathrm{MCT}^{+/+}$and $\mathrm{MCT}^{-1-}$ mice. We applied repeated sciatic nerve stimulations with increasing frequencies and recorded the evoked electromyographic (EMG) signal in the gastrocnemius muscle (Figure 7C). No difference was found in the amplitude decrement after $30 \mathrm{~s}$ of repetitive stimulations (data not shown). CMAP amplitude, however, expressed as root mean squared (RMS), was significantly lower in $\mathrm{MCT}^{-1-}$ at all frequencies tested (Figure 7D). Interestingly, loss of NMJ integrity and decreased CMAP amplitude have been described in patients with CMT (Elbracht et al., 2014) (Spaulding et al., 2016) (Cipriani et al., 2018). Together, these data suggest that ablation of MCT4 had a negative effect on the structural and functional stability of the motor unit.

\section{DISCUSSION}

In the current study, we investigated whether disruption of the lactate shuttle had an effect on exercise capacity, by characterizing the phenotype of the $\mathrm{MCT}^{-1-}$ mouse. The results of our study showed that mice with a global knockout of MCT4 had defects in locomotion both when tested for spontaneous in-cage activity (wheel running) and forced treadmill exercise (Figure 2). The decrement in motor function was more evident in the spontaneous activity after the mice reached the adult age, suggesting that the phenotype is progressive, and exacerbated during forced endurance test (Figure 2C). By combining the results from the $\mathrm{MCT}_{4}^{-1-}$ mice with those obtained from the $\mathrm{iMSBsg}^{-/-}$, we can conclude that the deletion of lactate transporters from the skeletal muscles is not detrimental for the muscle itself. Instead, the exercise intolerance observed is accompanied by a functional and structural impairment of the neuronal component of the motor unit. Although we cannot exclude that lack of MCT4 in the CNS affects behavior, the integration of in vivo and ex vivo functional assessments with morphological analyses suggests that $\alpha$ motor neurons and NMJs are mostly affected.

For decades, lactate was considered the cause of fatigue in exercising muscles based on experiments conducted in the 1920s on non-oxygenated frog muscles (Hill et al., 1924). The lactate shuttle hypothesis was described over 30 years ago by Brooks (Brooks, 1986), who suggested that lactate is a substrate exchanged between glycolytic and oxidative tissues/cells, allowing for the control of its cytosolic concentration. During the past 20 years, researchers in the muscle and exercise physiology fields have worked to understand the real effects of lactate and $\mathrm{H}^{+}$during muscle activity (Allen et al., 2008; Azevedo et al., 2007). It has now been established that lactate is not cause of fatigue (Posterino et al., 2001). The fate of the $\mathrm{H}^{+}$ions, which are cotransported together with lactate during fatiguing muscle contraction (Kemp et al., 2006; Robergs et al., 2004; Robergs, 2017), however, is still not clear. In our study we showed that in ex vivo electrically stimulated $\mathrm{MCT}^{-1-}$ muscles, despite a 2-fold accumulation of intramuscular lactate, there was no decrement in force generation or fatigability (Figure 4), supporting the fact that increased lactate was not the cause of fatigue. We also tested whether addition of exogenous L-lactate in the bath during the fatigue test could alter muscle performance, but no difference was detected (data not shown).

We used Bsg floxed mice to indirectly knock out both transporters in adult muscle, with the goal of testing whether it would have a detrimental impact on exercise capacity. Mice ubiquitously lacking Bsg are reported to be sterile and with memory and learning deficits (Naruhashi et al., 1997), but their exercise capacity has not been investigated. To our surprise, there was no difference between controls and $\mathrm{iMSBsg}^{-1-}$ in terms of running capacity or ex vivo contractility. This result is in contrast to the lactate shuttle hypothesis and more recent work, which suggest that lactate derived from skeletal muscle is a key metabolite utilized by different tissues as energy substrate (Hui et al., 2017). We cannot exclude that the lack of both transporters and their binding partner, BSG, may have caused a metabolic reprogramming in the skeletal muscle to limit lactate production and utilization. Taken together, the results from the two mouse models show that the systemic redistribution of muscle-derived lactate, operated by MCT1 and MCT4, can be bypassed during exercise.

Since its conception, the lactate shuttle theory has been applied to other systems such as cancer and neuroenergetics. Pellerin and Magistretti (Pellerin et al., 1998) presented the astrocytes-neurons lactate shuttle (ANLS) hypothesis where, in situations of high energy demand, astrocytes are a source of lactate and 
neurons are lactate consumers. Several studies have shown the expression of MCT4 in astrocytes in brain cortex and hippocampus (Bergersen et al., 2001) (Rafiki et al., 2003). In this study, we investigated the presence of MCT4 in the spinal cord and the potential role of lactate in supporting motor neuron activity. The in situ hybridization data (Figure 6B) showed that MCT4 is expressed by multiple cell types, but we did not confirm the expression in any particular cell type. Based on information available from the Allen Brain Atlas, as well as the topology of our in situ mRNA staining, we suggest that motoneurons are among the cells expressing MCT4. This is surprisingly new since previous reports indicated that MCT4 is expressed only in astrocytes. The neuronal MCT4 expression, in contrast with the ANLS hypothesis, is, however, supported by several publications suggesting that neurons rely on glycolytic metabolism for energy production, when stimulated (Gjedde and Marrett, 2001; Simpson et al., 2007) (Mangia et al., 2009) (Díaz-García et al., 2017). Future investigations will need to address if MCT4 and lactate have roles in the viability of the motor neuron and whether, as seen in several cancer studies (Marchiq et al., 2015), the blockade of lactate flux induces senescence and impairs neuronal signaling.

By performing CMAP measurements on $\mathrm{MCT}^{-1-}$ mice, we found a decrement in the amplitude of the EMG signal upon direct stimulation (Figure 7). Lower CMAP amplitude is a hallmark of axonal neuropathies such as CMT, multiple sclerosis (MS), and other pathologies of the NMJ. Despite the clear functional decline of the motor capacity (Figure 2) and the lower CMAPs amplitude in young adult mice, we did not detect any evident structural change in the sciatic nerve (Figure 6). This is consistent with the normal latency estimated during our CMAP experiments in both young adult and old mice (Manganelli et al., 2016) (Cornett et al., 2016).

Structural disruption of NMJs was evident in older mice (Figure 7), when the decline in spontaneous activity is also more pronounced. The results suggested that MCT4 and lactate may have an effect on the long-term integrity of the motor unit and NMJs. It is interesting to note that the degeneration of the NMJs was observed only in EDL muscles, which express MCT4 at the sarcolemma, and not in soleus muscles, which express mainly MCT1. Based on our results, we speculate that lactate released by the skeletal muscle may be necessary for the stability of the NMJs either functioning as metabolic substrate or as signaling molecule. A variety of factors as myokines and neurotrophic factors are important for the structural and functional integrity of the NMJ (Lin et al., 2008, 2001). Indeed, exercise has been shown to induce remodeling and adaptation of the NMJs (Deschenes et al., 2019), and lack or reduced muscle activity can have a detrimental effect on NMJ structure and function as seen during aging (Gonzalez-Freire, 2014).

It has been reported that MCT4 is expressed by Schwann cells of the peripheral nervous system (PNS) (Domènech-Estévez et al., 2015), where it might have a role in the maintenance of axon myelination. In our model, we did not detect the expression of MCT4 mRNA or protein in the PNS and we also did not observe any defect in axons myelination (Figure 6).

In conclusion, the results of our study show that the exercise intolerance observed in the $\mathrm{MCT}^{-/-}$mice is not attributable to disruption of the lactate shuttle between fast-twitch and slow-twitch muscle fibers. Rather, in the $\mathrm{MCT}_{4}^{-/-}$mice there is an instability of the motor unit and, in particular, a progressive degeneration of the NMJs. We propose that the lack of MCT4 from motoneurons decreases their activity possibly by altering the coordinate action of the metabolic pathways responsible for ATP production. This may in turn affect the release of neurotransmitters at the NMJs, hence the decreased amplitude of the CMAPs recorded. Therefore, the decreased overall activity of the $\mathrm{MCT} 4^{-1-}$ mice is associated with instability and premature degeneration of the NMJs. We suggest that the phenotype of $\mathrm{MCT4}^{-/-}$mice is reminiscent of neurodegenerative diseases such as Charcot-Marie-Tooth, Multiple Sclerosis, or ALS, and therefore it would be interesting to evaluate the role of MCT4 in these pathologies.

\section{Limitations}

In the current study we found that the lack of MCT4 negatively affects the exercise capacity of the mice but it had no impact on ex vivo muscle physiology. $\mathrm{MCT}^{-/-}$mice did exhibit a decrease in amplitude of the CMAPs accompanied by structural changes at the levels of the NMJs. Although our data suggest that the exercise intolerance is due to instability of the motor unit, our approach did not have sufficient resolution to determine whether the functional impairment of the NMJs is a cause or a consequence of exercise intolerance. In addition, we could only detect MCT4 transcripts in the spinal cord since the immunostaining to demonstrate the presence of the protein was not reliable because was giving a high non-specific signal 
also in the $\mathrm{MCT4}^{-1-}$ samples. We could not ascertain whether these effects resulted from lack of MCT4 expression in the motoneurons or astrocytes. In this study, owing to limited technology, we could not show whether the lack of MCT4 had a direct effect on neurotransmitter release from the pre-synapses or impaired activation of the motoneurons. Future studies using motoneurons and/or astrocytes MCT4 conditional knockout mice will help elucidate this important point.

\section{METHODS}

All methods can be found in the accompanying Transparent Methods supplemental file.

\section{SUPPLEMENTAL INFORMATION}

Supplemental Information can be found online at https://doi.org/10.1016/j.isci.2019.11.041.

\section{ACKNOWLEDGMENTS}

We thank the Bioimaging Shared Resource of the Sidney Kimmel Cancer Center (NCI 5 P30 CA-56036) and the Penn Muscle Institute Muscle Physiology Assessment Core for muscle contraction study. This work was primarily supported by the US National Institute of Health grants R01EY012042 to N.J.P. and R01NS079702 and R01NS110385 to A.C.L.

\section{AUTHOR CONTRIBUTIONS}

S.B, E.L., and N.J.P. designed and developed project and wrote the manuscript. S.B. and E.L. performed experiments and analyzed data. M.C.W. performed NMJ analysis. R.A.N. provided Bsg ${ }^{\text {flox/flox }}$ mice. A.C.L. and T.S.K. contributed with reagents and discussion. All the authors read and reviewed the manuscript.

\section{DECLARATION OF INTERESTS}

The authors declare no competing interests.

Received: July 8, 2019

Revised: October 30, 2019

Accepted: November 22, 2019

Published: December 20, 2019

\section{REFERENCES}

Adijanto, J., and Philp, N.J. (2012). The SLC16A family of monocarboxylate transporters (MCTs)physiology and function in cellular metabolism, $\mathrm{pH}$ homeostasis, and fluid transport. Curr. Top. Membr. 70, 275-311.

Allen, D.G., Lamb, G.D., and Westerblad, H. (2008). Skeletal muscle fatigue: cellular mechanisms. Physiol. Rev. 88, 287-332.

Asp, M.L., Martindale, J.J., and Metzger, J.M. (2013). Direct, differential effects of tamoxifen, 4-hydroxytamoxifen, and raloxifene on cardiac myocyte contractility and calcium handling. PLoS One 8, e78768

Azevedo, J.L., Tietz, E., Two-Feathers, T., Paull, J., and Chapman, K. (2007). Lactate, fructose and glucose oxidation profiles in sports drinks and the effect on exercise performance. PLoS One 2, e927

Bergersen, L., Waerhaug, O., Helm, J., Thomas, M., Laake, P., Davies, A.J., Wilson, M.C., Halestrap, A.P., and Ottersen, O.P. (2001). A novel postsynaptic density protein: the monocarboxylate transporter MCT2 is colocalized with delta-glutamate receptors in postsynaptic densities of parallel fiber-Purkinje cell synapses. Exp. Brain Res. 136, 523-534.
Bonen, A. (2001). The expression of lactate transporters (MCT1 and MCT4) in heart and muscle. Eur. J. Appl. Physiol. 86, 6-11.

Brooks, G.A. (1986). The lactate shuttle during exercise and recovery. Med. Sci. Sports Exerc. 18, 360-368

Cipriani, S., Phan, V., Médard, J.-J., Horvath, R., Lochmüller, H., Chrast, R., Roos, A., and Spendiff, S. (2018). Neuromuscular junction changes in a mouse model of Charcot-Marie-Tooth disease type 4C. Int. J. Mol. Sci. 19, 4072.

Connett, R.J., Honig, C.R., Gayeski, T.E., and Brooks, G.A. (1990). Defining hypoxia: a systems view of VO2, glycolysis, energetics, and intracellular PO2. J. Appl. Physiol. 68, 833-842.

Cornett, K.M.D., Menezes, M.P., Bray, P., Halaki, M., Shy, R.R., Yum, S.W., Estilow, T., Moroni, I.,

Foscan, M., Pagliano, E., et al. (2016). Phenotypic variability of childhood Charcot-Marie-Tooth disease. JAMA Neurol. 73, 645-651.

Desai, K.H., and Bernstein, D. (2001). Exercise and oxygen consumption in the mouse. In Cardiovascular Physiology in the Genetically Engineered Mousee. Developments in Cardiovascular Medicine, 238, B.D. Hoit and R.A Walsh, eds., Cardiovascular Physiology in the
Genetically Engineered Mousee. Developments in Cardiovascular Medicine (Springer), pp. 277-302.

Deschenes, M.R., Tufts, H.L., Noronha, A.L., and $\mathrm{Li}, \mathrm{S}$. (2019). Both aging and exercise training alter the rate of recovery of neuromuscular performance of male soleus muscles. Biogerontology 20, 213-223.

Dimmer, K.S., Friedrich, B., Lang, F., Deitmer, J.W., and Bröer, S. (2000). The low-affinity monocarboxylate transporter MCT4 is adapted to the export of lactate in highly glycolytic cells. Biochem. J. 350 Pt 1, 219-227.

Díaz-García, C.M., Mongeon, R., Lahmann, C. Koveal, D., Zucker, H., and Yellen, G. (2017).

Neuronal stimulation triggers neuronal glycolysis and not lactate uptake. Cell Metab. 26, 361374.e4.

Domènech-Estévez, E., Baloui, H., Repond, C. Rosafio, K., Médard, J.-J., Tricaud, N., Pellerin, L., and Chrast, R. (2015). Distribution of monocarboxylate transporters in the peripheral nervous system suggests putative roles in lactate shuttling and myelination. J. Neurosci. 35, 41514156 
Elbracht, M., Senderek, J., Schara, U., Nolte, K., Klopstock, T., Roos, A., Reimann, J., Zerres, K., Weis, J., and Rudnik-Schöneborn, S. (2014). Clinical and morphological variability of the E396K mutation in the neurofilament light chain gene in patients with Charcot-Marie-Tooth disease type 2E. Clin. Neuropathol. 33, 335-343.

Gjedde, A., and Marrett, S. (2001). Glycolysis in neurons, not astrocytes, delays oxidative metabolism of human visual cortex during sustained checkerboard stimulation in vivo. J. Cereb. Blood Flow Metab. 21, 1384-1392.

Gonzalez-Freire, M. (2014). The neuromuscular junction: aging at the crossroad between nerves and muscle. Front. Aging Neurosci. 6, 208.

Hill, A.V., Long, C.N.H., and Lupton, H. (1924) Muscular exercise, lactic acid and the supply and utilisation of oxygen. Proc. R. Soc. B: Biol. Sci. 97 155-176.

Hill, M. (2003). The neuromuscular junction disorders. J. Neurol. Neurosurg. Psychiatry 74 (Suppl 2), ii32-ii37.

Hui, S., Ghergurovich, J.M., Morscher, R.J., Jang C., Teng, X., Lu, W., Esparza, L.A., Reya, T., Le Zhan, Guo, J.Y., et al. (2017). Glucose feeds the TCA cycle via circulating lactate. Nature 551, 115-118.

Juel, C. (1997). Lactate-proton cotransport in skeletal muscle. Physiol. Rev. 77, 321-358.

Kemp, G., Böning, D., Beneke, R., and Maassen, N. (2006). Explaining pH change in exercising muscle: lactic acid, proton consumption, and buffering vs. strong ion difference. Am. J. Physiol. Regul. Integr. Comp. Physiol. 291, R235-R237. [author reply R238-9].

Kirk, P. Wilson, M.C. Heddle, C., Brown, M.H. Barclay, A.N., and Halestrap, A.P. (2000). CD147 is tightly associated with lactate transporters MCT1 and MCT4 and facilitates their cell surface expression. EMBO J. 19, 3896-3904.
Lin, S., Landmann, L., Ruegg, M.A., and Brenner, H.R. (2008). The role of nerve- versus musclederived factors in mammalian neuromuscular junction formation. J. Neurosci. 28, 3333-3340.

Lin, W., Burgess, R.W., Dominguez, B., Pfaff, S.L. Sanes, J.R., and Lee, K.F. (2001). Distinct roles of nerve and muscle in postsynaptic differentiation of the neuromuscular synapse. Nature 410, 10571064

Manganelli, F., Pisciotta, C., Reilly, M.M., Tozza, S., Schenone, A., Fabrizi, G.M., Cavallaro, T., Vita G., Padua, L., Gemignani, F., et al. (2016). Nerve conduction velocity in CMT1A: what else can we tell? Eur. J. Neurol. 23, 1566-1571.

Mangia, S., Simpson, I.A., Vannucci, S.J., and Carruthers, A. (2009). The in vivo neuron-toastrocyte lactate shuttle in human brain: evidence from modeling of measured lactate levels during visual stimulation. J. Neurochem. 109 (Supp/ 1), $55-62$

Marchiq, I., Le Floch, R., Roux, D., Simon, M.-P., and Pouyssegur, J. (2015). Genetic disruption of lactate/H+ symporters (MCTs) and their subunit CD147/BASIGIN sensitizes glycolytic tumor cells to phenformin. Cancer Res. 75, 171-180.

Naruhashi, K., Kadomatsu, K., Igakura, T., Fan, Q.-W., Kuno, N., Muramatsu, H., Miyauchi, T., Hasegawa, T., Itoh, A., Muramatsu, T., et al. (1997). Abnormalities of sensory and memory functions in mice LackingBsgGene. Biochem. Biophys. Res. Commun. 236, 733-737.

Pellerin, L., Pellegri, G., Bittar, P.G., Charnay, Y. Bouras, C., Martin, J.L., Stella, N., and Magistretti, P.J. (1998). Evidence supporting the existence of an activity-dependent astrocyte-neuron lactate shuttle. Dev. Neurosci. 20, 291-299.

Philp, N.J., Ochrietor, J.D., Rudoy, C., Muramatsu, T., and Linser, P.J. (2003). Loss of MCT1, MCT3, and MCT4 expression in the retinal pigment epithelium and neural retina of the
5A11/basigin-null mouse. Invest. Ophthalmol. Vis. Sci. 44, 1305-1311.

Poole, R.C., and Halestrap, A.P. (1993). Transport of lactate and other monocarboxylates across mammalian plasma membranes. Am. J. Physiol. 264, C761-C782.

Posterino, G., Dutka, T., and Lamb, G. (2001). L (+)-lactate does not affect twitch and tetanic responses in mechanically skinned mammalian muscle fibres. Pflugers Arch. 442, 197-203.

Rafiki, A., Boulland, J.L., Halestrap, A.P. Ottersen, O.P., and Bergersen, L. (2003). Highly differential expression of the monocarboxylate transporters MCT2 and MCT4 in the developing rat brain. Neuroscience 122, 677-688.

Robergs, R.A., Ghiasvand, F., and Parker, D. (2004). Biochemistry of exercise-induced metabolic acidosis. Am. J. Physiol. Regul. Integr. Comp. Physiol. 287, R502-R516.

Robergs, R.A. (2017). Competitive cation binding computations of proton balance for reactions of the phosphagen and glycolytic energy systems within skeletal muscle. PLoS One 12, e0189822

Simpson, I.A., Carruthers, A., and Vannucci, S.J. (2007). Supply and demand in cerebral energy metabolism: the role of nutrient transporters. J. Cereb. Blood Flow Metab. 27, 1766-1791.

Spaulding, E.L., Sleigh, J.N., Morelli, K.H., Pinter, M.J., Burgess, R.W., and Seburn, K.L. (2016). Synaptic deficits at neuromuscular junctions in two mouse models of charcot-marie-tooth type 2d. J. Neurosci. 36, 3254-3267.

Wilson, M.C., Jackson, V.N., Heddle, C., Price N.T., Pilegaard, H., Juel, C., Bonen, A., Montgomery, I., Hutter, O.F., and Halestrap, A.P. (1998). Lactic acid efflux from white skeletal muscle is catalyzed by the monocarboxylate transporter isoform MCT3. J. Biol. Chem. 273 15920-15926. 
ISCI, Volume 22

Supplemental Information

New Insights into the Lactate

Shuttle: Role of MCT4 in the Modulation

of the Exercise Capacity

Sara Bisetto, Megan C. Wright, Romana A. Nowak, Angelo C. Lepore, Tejvir S.

Khurana, Emanuele Loro, and Nancy J. Philp 


\section{Figure S1}

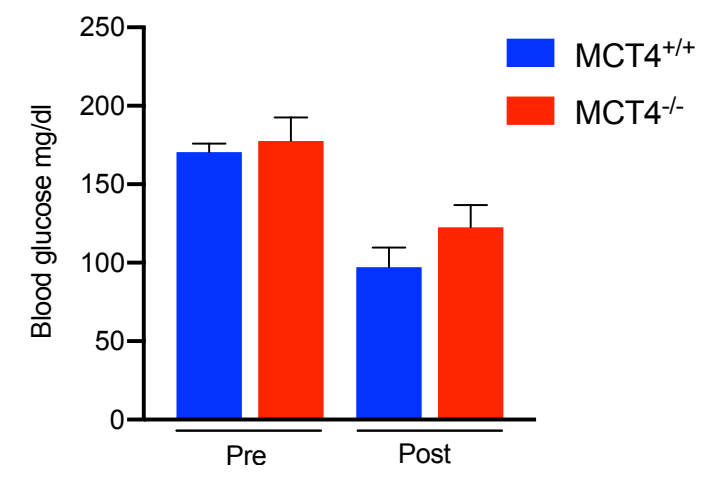

Figure S1, related to figure 2: Blood glucose measurement before and after treadmill test: (A) Venus blood glucose measured in 6 months old $\mathrm{MCT}^{+/+}$and $\mathrm{MCT}^{-/-}$ before and after treadmill test, $(\mathrm{n}=5$ each group). Error bars $=$ SEM. 
Figure S2

A

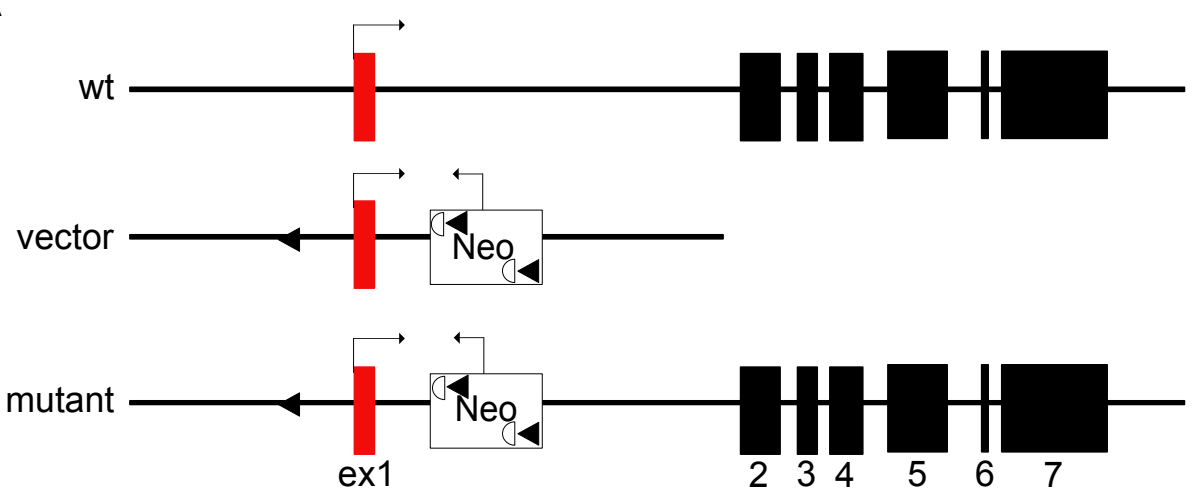

lexon 14 lox P $\bigcirc$ rt

B

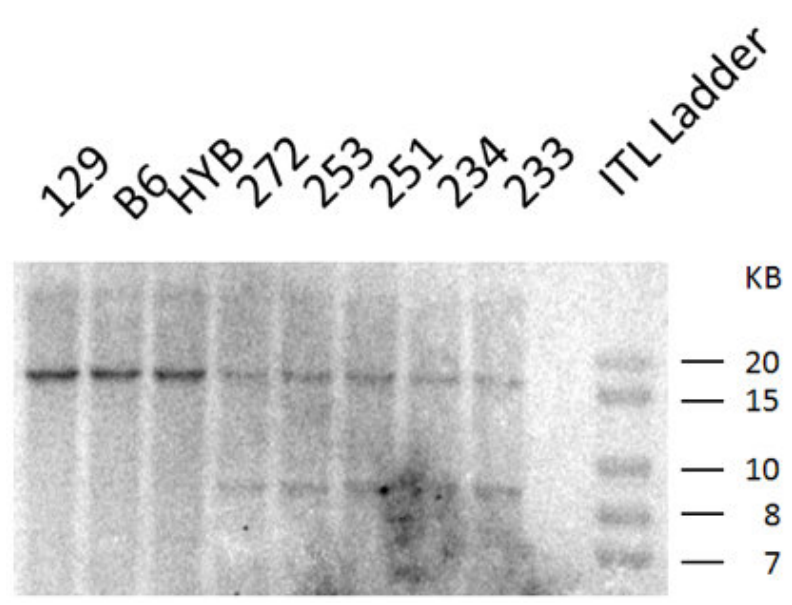

Figure S2, related to figure 5: Generation of the Bsg conditional mice. (A) Design of targeting vector to produce floxed Bsg mouse with placement of LoxP sites to target exon 1 of Bsg gene for deletion. (B) Southern blot confirmation of five ES clones (233, $234,251,253$ and 272) as correctly targeted and used for injection. 
Figure S3

A

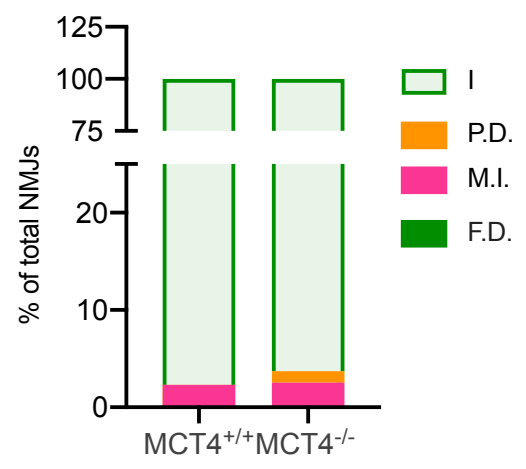

B

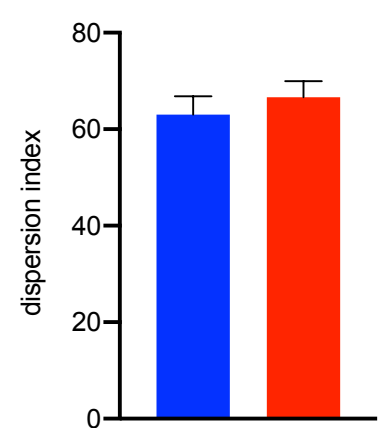

C $\quad 12$ months

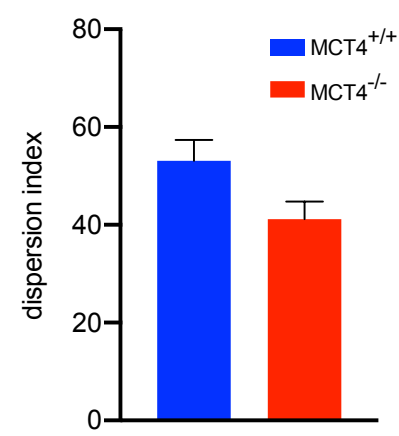

Fig S3, related to figure 7: Morphological analysis of NMJs in soleus muscles and postsynaptic endplate: (A) Quantification of intact and disrupted NMJs in soleus muscles of 12 months old MCT4 ${ }^{-/-}$and MCT4 $4^{+/+}(n=3$ each group). Quantification of the density of Acetylcholine receptors (AChR) at the postsynaptic endplate from EDLs cross sections from (B) 6 months old ( $n=4$ each group, 6 NMJs per animal) and (C) 12 months old $\left(n=3\right.$ each group, 3 NMJs per animal) $\mathrm{MCT}^{+/+}$and MCT4 ${ }^{-/-}$mice. Error bars $=$SEM. 
Table S1 related to Figure 4: Contractile and morphometric measurements of EDL muscles.

\begin{tabular}{|l|c|c|c|}
\hline & MCT4 $^{+/+}$ & MCT4 $^{-/-}$ & $P$ \\
\hline EDL contractility & $\mathrm{N}=4$ & $\mathrm{~N}=5$ & \\
\hline Twitch force $(\mathrm{mN})$ & $94.68 \pm 13.90$ & $118.22 \pm 4.71$ & 0.121 \\
\hline Twitch force $\left(\mathrm{N} / \mathrm{cm}^{2}\right)$ & $5.36 \pm 0.62$ & $6.04 \pm 0.13$ & 0.265 \\
\hline Tetanic force $(\mathrm{mN})$ & $345.08 \pm 53.87$ & $447.76 \pm 7.18$ & 0.070 \\
\hline Tetanic force $\left(\mathrm{N} / \mathrm{cm}^{2}\right)$ & $19.42 \pm 2.10$ & $22.95 \pm 0.57$ & 0.115 \\
\hline Twitch - TTP $(\mathrm{ms})$ & $20 \pm 0.42$ & $20.10 \pm 0.27$ & 0.841 \\
\hline Twitch - RFD $(\mathrm{mN} / \mathrm{sec})$ & $12747.25 \pm 1909.27$ & $14428.00 \pm 892.54$ & 0.419 \\
\hline Fatigue AUC & $956.90 \pm 83.52$ & $981.38 \pm 82.61$ & 0.843 \\
\hline & & & \\
\hline EDL morphometry & $\mathrm{N}=4$ & $\mathrm{~N}=5$ & \\
\hline Lo $(\mathrm{mm})$ & $11.93 \pm 0.37$ & $12.56 \pm 0.10$ & 0.106 \\
\hline Mass $\left(\mathrm{mg}^{2}\right)$ & $9.98 \pm 0.63$ & $11.74 \pm 0.52$ & 0.066 \\
\hline CSA $\left(\mathrm{mm}^{2}\right)$ & $1.76 \pm 0.12$ & $1.96 \pm 0.08$ & 0.191 \\
\hline
\end{tabular}

Values are mean \pm SEM. TTP, time to peak; RFD, rate of force development; CSA, cross sectional area; Lo, muscle length. 


\section{TRANSPARENT METHODS}

Animals.

All animal procedures were performed in accordance with the Thomas Jefferson University Institutional Animal Care and Use Committee. MCT4 (s/c16a3) knock out mice, stock no. TF3223A, were obtained from Taconic Farms. Mice were backcrossed for 10 generations to C57BL/6NTac to obtain a $99 \%$ C57BL/6 background. Bsg flox/flox mice were obtained from Dr. Nowak at University of Illinois. The mice were generated by inGenious Targeting Laboratory (www. Genetargeting.com). The construct was designed to target exon 1 for conditional deletion as shown in the supplementary material (Fig. S1A). Briefly, ten micrograms of the targeting vector were linearized by Notl and then transfected by electroporation of BA1(C57BI/6 x 129/SvEv) (Hybrid) embryonic stem cells. After selection with G418 antibiotic, surviving clones were expanded for PCR analysis and southern blotting to identify recombinant ES clones. Sequencing was also performed on purified PCR DNA to confirm the presence of the LoxP cassette using the SDL2 primer. Five positive clones were confirmed by southern blotting (Fig. S1B) and used for blastocyst injection, implantation and generation of chimeras. Germline transmission was confirmed in four of the clones. Bsg flox/flox were crossed with tamoxifen inducible Acta1cre/Esr1 mice (Jackson Lab, stock no 025750 HSA-MCM). Induction of the cre-recombinase was obtained by daily injection of tamoxifen $(2 \mathrm{mg} / \mathrm{kg})$, for 5 consecutive days. Bsg ${ }^{\text {flox/flox:Cre }}{ }^{+/-}$mice injected with vehicle $\left(15 \%\right.$ ethanol in sunflower oil) were used as control. Mice were housed at $22^{\circ} \mathrm{C}$ and under $12: 12 \mathrm{~h}$ light-dark cycle. Male mice were used in all the reported experiments except for the reported wheels running test and NMJs morphology data.

\section{Histology.}

Tibialis anterioris (TA) muscle and extensor digitorum longus (EDL) muscles were frozen in Tissue-Tek OCT compound (WVR, Atlanta GA) and stored at $-80^{\circ} \mathrm{C}$. $10 \mu \mathrm{m}$ cryosections were cut and used for subsequent histological and immunofluorescence stainings. For H\&E staining, a standard procedure was followed. For fiber typing analysis, sections were stained with monoclonal antibodies against myosin heavy chains produced by Dr Schiaffino's lab at University of Padova and distributed by the Developmental Studies Hybridoma Bank (DHSB, University of lowa). Ba-D5 (supernatant), specific for MyHC-1 was used at 1:100 dilution, Bf-F3 (purified antibody), specific for MyHC-IIB was used at 1:100 and Sc-71 (supernatant), specific for MyHC-IIA, was used at 1:50. The secondary antibodies: donkey anti-mouse IgG2b AlexaFluor647 to bind Ba-D5; donkey anti-mouse IgM AlexaFluor 555 to bind Bf-F3 and donkey anti-mouse lgG1 AlexaFluor 488 to bind Sc-71 (Life Technologies, New York) were diluted 1:500. Membranes were counterstained with anti-laminin antibody (L-9393, Sigma) or wheat germ agglutinin (WGA) conjugated with AlexaFluor 488 or AlexaFluor 647 (Thermo Fisher). Images were collected using the epifluorescence microscope Eclipse 800 (Nikon) and analysis of the distribution of the different fiber types were performed with the ImageJ software. For the analysis of MCT1 and MCT4 distribution, anti-MCT1 and anti-MCT4 antibodies previously characterized by our laboratory (Philp et al., 2003) were used. For spinal cord histological analysis, samples were collected and fixed overnight in $4 \%$ paraformaldehyde. After cryopreservation in 30\% sucrose solution the samples were frozen in Tissue-tek OCT compound and stored at -80. For motoneurons analysis, 25um thick transversal sections from the lumbar portion of the spinal cord were cut and stained with $0.5 \%$ cresyl violet acetate solution and images were captured using cellSens Entry software and the Olympus DP22 camera attached to an Olympus CX41 microscope (Olympus Scientific Solutions Americas). Motoneurons were identified as larger polygonal cells, with diameter larger than $100 \mu \mathrm{m}$ in the ventral horn. For every subject 3 different sections, $150 \mu \mathrm{m}$ apart were evaluated.

\section{Exercise tolerance test.}

Exercise tolerance was assessed using a motorized Exer3-6 treadmill system with shock counter (Columbus Instruments, Columbus $\mathrm{OH}$ ). Mice were acclimated to the treadmill for 3 consecutive days with a 10 minutes session at 0 incline (1- zero shock, $5 \mathrm{~m} / \mathrm{min}$ speed, 2 - $1 \mathrm{hz} 0.5 \mathrm{~mA}$ shock, $5 \mathrm{~m} / \mathrm{min}$ speed for $5 \mathrm{~min}, 10 \mathrm{~m} / \mathrm{min}$ speed $5 \mathrm{~min}$, 3- $1 \mathrm{hz} 0.5 \mathrm{~mA}$ shock, $10 \mathrm{~m} / \mathrm{min}$ speed). Between acclimation and test the mice rested for $48 \mathrm{~h}$. The test was performed at $5^{\circ}$ incline, it started at $10 \mathrm{~m} / \mathrm{min}$ and the speed was increased by $2 \mathrm{~m} / \mathrm{min}$ every 3 minutes, until a maximum speed of $26 \mathrm{~m} / \mathrm{min}$ was reached. Maximum speed, time on belt and distance run, as well as the number of the shocks received during the test, were recorded. The mice were considered exhausted when they sat on the shock grid for 5 consecutive seconds despite the shocks. 
Voluntary wheel running test.

Mice were single housed in cages equipped with wheels (Columbus Instrument) for 7 days. Wheel revolutions during the last 60 hours were acquired and analyzed.

\section{RNA analysis.}

RNA was extracted with TRIzol Reagent (Thermo Fisher) and cDNA synthesis was performed using the RNA to cDNA EcoDry ${ }^{\mathrm{TM}}$ Premix kit (Clontech). SYBR green qPCR analysis was performed on the QuantStudio 5 real time system (Thermo Fisher). Relative expression was calculated using $R p / p 0$ as housekeeping. The primers used were:

Car2: Fw CAGCGAGCAGATGTCTCATTTC, Rev TTCTTTAGCGGCTGAGCTGG, Car3: Fw TTAAGACGAAGGGCAAGGAGG, Rev AGGAGCCGTGATAGGTCCAA, Car4: Fw GAGCCAGCTATCCACGTTCC, Rev TTCCGGTCTGCTCTGCCTAT, NEH1: Fw GCCTCATGAAGATAGGTTTCCA, Rev ACGTCTGATTGCAGGAAGGG, MCT1: Fw TGTTAGTCGGAGCCTTCATT, Rev CACTGGTCGTTGCACTGAATA, Rplp0: Fw: AGATTCGGGATATGCTGTTGGC, Rev TCGGGTCCTAGACCAGTGTTC.

\section{Intramuscular lactate measurement.}

After stimulation, muscles were rapidly frozen in liquid nitrogen. 30 to $50 \mathrm{mg}$ of gastrocnemius muscle were homogenized in ice cold $0.6 \mathrm{M}$ perchloric acid with a handheld homogenizer and then centrifuged at $14000 \mathrm{rpm}$ for 15 minutes. The supernatant $\mathrm{pH}$ was neutralized with $1.8 \mathrm{M}$ potassium carbonate. After further centrifugation the supernatants were collected and used for lactate assay using a commercial kit (Trinity Biotech).

\section{Blood Glucose measurement.}

Blood glucose was measured before and right after treadmill test from tail blood using a commercialy available glucose meter (Precision Xtra Glucose Meter, Abbot)

\section{Western Blot analysis.}

Skeletal muscle tissue was homogenized in a buffer containing $50 \mathrm{mM}$ Tris, pH8, $150 \mathrm{mM} \mathrm{NaCl}, 5 \mathrm{mM}$ EDTA, $0.1 \%$ SDS, $1 \%$ Triton X-100 and 0.5\% Deoxycholic acid. Samples were separated in a $4-12 \%$ Bis-Tris precast Nupage gels (Thermo fisher) and blotted onto PVDF membranes. Membranes were blocked in 5\% no-fat milk in TBS-tween buffer and incubated overnight with primary antibodies. After washing the membranes in TBS-tween buffer they were incubated in HRP conjugated secondary antibodies for $1 \mathrm{~h}$ at room temperature. We developed the blots using SuperSignal West Dura ECL substrate (ThermoFisher Scientific) and imaged using FluorChem M ProteinSimple imager.

\section{CMAP and ex vivo EDL contractility analysis.}

During all ex vivo and in vivo procedures, the genotype of the mice was not disclosed to the operator. For the analysis of Compound Muscle Action Potential (CMAP), mice were anesthetized with $1 \%$ isoflurane. Depth of anesthesia was confirmed by toe pinch and by monitoring heart rate and $\mathrm{SpO}_{2}$ with a pulse oximeter (PhysioSuite, Kent Scientific). The sciatic nerve was exposed, crushed distally to the muscle to prevent retrograde propagation of the stimuli, and connected to a custom-made bipolar stimulating electrode driven by a Grass S48 stimulator. Two recording silver EMG needle-electrodes were applied at fixed distance $(5 \mathrm{~mm})$, one in the gastrocnemius and the other near the Achilles tendon. The ground electrode was connected to the skin of the back of the mouse. The recording electrodes were then connected to a Warner Instruments DP-311 differential amplifier, band-pass filtered (0.1-10k $\mathrm{Hz}$ ) and finally fed into a PowerLab/8Sp A/D converter (AD Instruments). One leg of each mouse was electrically stimulated, while the other was left unstimulated and used to dissect the EDL muscle for ex vivo contractility testing after completion of the CMAP protocol. Three trains of ten stimulating pulses ( $5 \mathrm{~V}, 0.1 \mathrm{~ms}$ duration) were applied for each frequency tested $(1,3,5,10,20,40 \mathrm{~Hz})$, allowing 2 minutes for recovery between each train. Data were acquired and analyzed with LabChart8 (AD Instruments), calculating EMG amplitude and root mean square (RMS) of the signal.

Muscle physiological analysis was performed on isolated EDL muscles using an Aurora Mouse 1200A System equipped with Dynamic Muscle Control v.5.415 software. EDL muscles were dissected and analyzed in constantly oxygenated Ringer's solution $(100 \mathrm{mM} \mathrm{NaCl}, 4.7 \mathrm{mM} \mathrm{KCl}, 3.4 \mathrm{mM} \mathrm{CaCl}, 1.2 \mathrm{mM}$ $\mathrm{KH}_{2} \mathrm{PO}_{4}, 1 . \mathrm{mM} \mathrm{MgSO}_{4}, 25 \mathrm{mM}$ HEPES, $5.5 \mathrm{mM}$ D-glucose) at $24^{\circ} \mathrm{C}$. The twitch stimulation protocol applied was a single stimulus with a duration of $0.2 \mathrm{~ms}$. Muscle length was adjusted to obtain the maximal twitch response and this length was measured and recorded as optimal length $\left(\mathrm{L}_{0}\right)$. For measuring tetanic maximal force generation, the stimulus was repeated at a frequency of $120 \mathrm{~Hz}$ for $500 \mathrm{~ms}$. Five minutes were allowed between two tetanic contractions to ensure muscle recovery. For 
induction of fatigue, $5 \mathrm{~min}$ after the last maximal tetanic contraction, muscles were stimulated every second for $8 \mathrm{~min}$ using $40-\mathrm{Hz}$ pulses lasting $330 \mathrm{~ms}$. Muscle cross-sectional area (CSA) of EDL muscles was calculated by dividing the muscle mass by the product of the muscle density coefficient ( 1.06 $\left.\mathrm{g} / \mathrm{cm}^{3}\right)$, muscle $\mathrm{L}_{0}$, and the fiber length coefficient $(0.45$ for $E D L)$. Specific force was determined by normalizing maximum isometric tetanic force to CSA.

\section{In situ hybridization.}

RNAScope technology was used for in situ hybridization following manufacturer's protocol. Briefly, 10um thick section were pretreated with hydrogen peroxide and protease. The slides were then incubated with RNAScope probe (mmSlc16a3) for $2 \mathrm{~h}$ at $40^{\circ} \mathrm{C}$ followed by the amplification steps according to protocol. Signal was developed using the RNAscope 2.5 HD Manual Detection Kit Red. The slides were then blocked in 5\% BSA and immunostained with anti GFAP (Sigma) over night. The next day donkey anti rabbit AlexaFluor 488 (Thermo Fisher) secondary antibody was applied for 1 hour and after washing slides were mounted in ProLong Diamond antifade mounting media with DAPI (Thermo Fisher). Images were captured on a Nikon Eclipse E800 with 4x/0.13 and 40x/0.75 DIC objectives.

\section{Sciatic nerve morphological analysis.}

Sciatic nerves were dissected and fixed in $4 \%$ glutaraldehyde in $0.1 \mathrm{M}$ Millonig buffer for 2 hours, rinsed in $0.54 \%$ glucose in Millonig buffer overnight and osmicated in $2 \% \mathrm{OsO}_{4}$ for 45 minutes. After dehydrations the samples were embedded in Durcupan resin. $500 \mathrm{~nm}$ sections were cut with a glass knife using a Leica UCT ultramicrotome, and then stained with $1 \%$ Toluidine blue and images were captured with Olympus CX41 microscope (Olympus Scientific Solutions Americas). Morphometric analysis of the myelinated axons was done with the Halo software, Axon module (IndicaLab, Albuquerque NM, USA). Axons and fibers diameter were recorded, and g-ratio was calculated.

\section{Neuromuscular junction staining.}

EDLs and solei were dissected, cleaned from connective tissue and fixed in 4\%PFA and processed for immunohistochemistry as previously described (Wright et al., 2007). They were then incubated with rhodamine-conjugated alpha-bungarotoxin (Thermo Fisher) to label acetylcholine receptors. After permeabilization in methanol, samples were blocked in 2\% BSA and then incubated overnight with primaries antibodies, SMI-312R (Covance) and SV-2 (Hybridoma bank) for labeling motor axons and terminal vesicles, respectively. After washing in PBS, samples were incubated for 1 hour with with FITC anti-mouse IgG secondary (Jackson ImmunoResearch). Samples were mounted with Vectashield mounting media and coverslipped. Samples were then analyzed for total numbers of intact, completely denervated, partially denervated and multiply innervated NMJs. Each junction was categorized as 1) intact if there was complete overlap between presynaptic nerve terminal and postsynaptic AChRs, 2) partially denervated with only partial occupation of postsynaptic AChRs by the overlying nerve terminal, or 3) completely denervated, with no occupation of postsynaptic AChRs by the nerve terminal. To ensure that a junction was completely denervated, and that the absence of the nerve terminal was not an artifact of the staining procedure, junctions were only counted as completely denervated if there were other nearby labeled junctions in the same viewing plane. Rapresentative images were captured using a Nikon A1R confocal microscope, with a 40X/1.3 oil objective. For analysis of the post synaptic endplate density, $25 \mu \mathrm{m}$ thick frozen cross sections of EDLs muscle were stained with rhodamineconjugated alpha-bungarotoxin. Z-stack images of en-face NMJs were collected using the Nikon A1R confocal microscope and processed with ImageJ to obtain a maximum intensity projection from the Zstack. Using the Cell Profiler software the images were batch processed and total area occupied by the staining and the perimeter were quantified. Dispersion index was calculated as ratio between the total perimeter and total area.

\section{Statistical analyses.}

Data are represented as mean \pm SEM. Data were processed and analyzed with GraphPad Prism 8 (GraphPad software). Before any statistical analysis was applied, data were assessed for normality using the D'Agostino-Pearson's test. Normally distributed data were compared using the student's $t$ test, for non-normlly distributed data, the Mann-Whitney test was applied. Data in Fig.2A were analyzed using a One-Way ANOVA test, followed by Tukey's test for multiple comparisons. Data in Fig.3E-F and 4D were analyzed with a 2-way ANOVA test followed by Tukey's test for multiple comparisons. For analysis of NMJs in Fig.7B a Chi-square test was applied. $\mathrm{P}<0.05$ was considered statistically significant.

\section{REFERENCES:}

Wright, M.C., Cho, W.-J., Son, Y.-J., 2007. Distinct patterns of motor nerve terminal sprouting induced by ciliary neurotrophic factor vs. botulinum toxin. J. Comp. Neurol. 504, 1-16. 